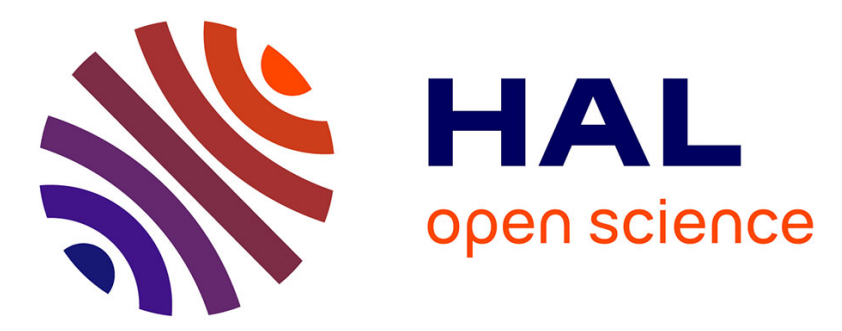

\title{
Revue de la boucle à re-modulation pour la synchronisation de phase en modulation linéaire multi-voie avec trajets multiples. Application en CDMA et OFDM.
}

Laurent Ros, Eric Pierre Simon, Youssef Nasser

\section{To cite this version:}

Laurent Ros, Eric Pierre Simon, Youssef Nasser. Revue de la boucle à re-modulation pour la synchronisation de phase en modulation linéaire multi-voie avec trajets multiples. Application en CDMA et OFDM.. La Revue de l'électricité et de l'électronique, 2005, 4, pp.55-68. hal-00275662

\section{HAL Id: hal-00275662 \\ https://hal.science/hal-00275662}

Submitted on 24 Apr 2008

HAL is a multi-disciplinary open access archive for the deposit and dissemination of scientific research documents, whether they are published or not. The documents may come from teaching and research institutions in France or abroad, or from public or private research centers.
L'archive ouverte pluridisciplinaire HAL, est destinée au dépôt et à la diffusion de documents scientifiques de niveau recherche, publiés ou non, émanant des établissements d'enseignement et de recherche français ou étrangers, des laboratoires publics ou privés. 


\title{
Revue de la boucle à re-modulation pour la synchronisation de phase en modulation linéaire multi-voie avec trajets multiples. Application en CDMA et OFDM.
}

\author{
Laurent Ros ${ }^{1}$, Eric Simon ${ }^{1}$, Youssef Nasser ${ }^{2}$ \\ $\{1\}$ Laboratoire des Images et des Signaux (LIS, UMR5083), Grenoble, \\ $\{2\}$ Laboratoire d'Electronique et de Technologies de l'Information, (CEA/LETI), Grenoble
}

\section{Résumé}

Cet article traite de la synchronisation de phase pour un récepteur radio-mobile en voie descendante, lorsque les trajets multiples du canal de propagation sont soumis à des variations de phases, mais avec des délais quasi-constants sur la durée d'un bloc de données (mode " burst"). On suppose un système assez général de modulation linéaire multi-voie, englobant notamment les cas des modulations CDMA et OFDM. Nous proposons une discussion générale du problème s'appuyant sur un formalisme par formes d'ondes. Pour cela, nous partons de la boucle à re-modulation ("Decision-Directed-Loop") standard utilisée en contexte mono-trajet et mono-utilisateur, et discutons de son utilisation en contexte à $L$ trajets et $K$ voies. L'extension de l'algorithme et les difficultés associées apparaissent ainsi de nature complètement différentes en CDMA et en OFDM, malgré un formalisme de modulation identique. Ceci est mis en évidence à l'aide d'illustrations s'appuyant sur les formes d'ondes spécifiques à chaque modulation (large-bande en CDMA, bande-étroite en OFDM mais avec une absence d'interférence préservée en présence de trajets multiples). Pour finir, nous présentons, pour le cas CDMA, une amélioration de la boucle standard, qui permet de fonctionner en présence de trajets non résolus, et de suivre de rapides variations de phases. L'amélioration consiste à insérer un pré-filtre dans la boucle de phase de chaque trajet, afin de corriger les formes d'ondes et rendre l'estimation indépendante (à retards constants) des autres trajets.

\section{Introduction}

Dans cet article (issu de la communication [8]), nous traitons de la synchronisation de phase au niveau du récepteur d'une voie descendante de radio-communication, lorsque les trajets multiples du canal de propagation sont soumis à des variations de phase, mais avec des délais quasi-constants sur la durée d'un bloc de données. Ce scénario correspond à une situation typique en communication radio-mobile par mode "Burst'. Par exemple, dans le mode TDD de l'UMTS, la fréquence porteuse est autour de $2 \mathrm{GHz}$, le débit "chip" $\frac{1}{T_{c}}$ est de l'ordre de $4 \mathrm{Mchip} / \mathrm{s}$ et le "slot" dure $T_{\text {slot }}=666 \mu \mathrm{s} . \mathrm{La}$ variation maximale possible de phase, " $360^{\circ} \Delta f_{d} T_{\text {slot }}$ ", est d'environ $55^{\circ}$ à $120 \mathrm{~km} / \mathrm{h}$, avec $\Delta f_{d}=\frac{v_{m}}{v_{0}} f_{0}$ l'étalement doppler, $v_{m}$ la vitesse du mobile et $v_{0}$ la célérité de l'onde. En ce qui concerne la variation maximale du retard de propagation, “ $\frac{v_{m}}{v_{0}} T_{\text {slot }}$ ”, on vérifie qu'elle est négligeable durant un "slot", de l'ordre de $3.10^{-4}$ par rapport à la durée d'un chip. On considère un système d'émission assez général, avec un débit global à transmettre au travers d'une largeur de bande $W$. On suppose que le débit global est partagé de manière uniforme en $K$ voies parallèles de symboles. A chaque voie (ou train numérique) est associée une forme d'onde particulière pour la mise en forme de ses symboles. Un tel formalisme de modulation linéaire englobe notamment les systèmes de type CDMA ou OFDM. La modulation considérée pour les symboles est de type QPSK. Nous ne traitons pas ici de la compensation des décalages de fréquence dus aux oscillateurs ni de l'estimation du retard de propagation (Cf [3]), que nous supposons préalablement réalisés dans une phase d'acquisition. Les objectifs de l'article sont:
- de décrire les techniques CDMA et OFDM à partir d'un formalisme commun de représentation par formes d'ondes. Cette représentation "analogique", certes assez éloigné de la réalisation effective des équipements, permet de mettre en évidence et d'interpréter facilement les effets du canal,

- de discuter de l'utilisation en contexte multi-trajet multi-voie, d'un algorithme de base standard [1] de poursuite de phase à l'aide des décisions (boucle à remodulation) conçu initialement pour un contexte mono-trajet et mono-utilisateur, avec application au cas CDMA et OFDM,

- de présenter, pour le cas CDMA, une amélioration possible [6] de l'algorithme standard qui permet de suivre de rapides variations des phases des trajets, tout en restant robuste vis à vis des trajets non résolus.

Après une description du modèle de la transmission puis de l'algorithme standard de synchronisation, nous étudierons son comportement en situation mono-trajet puis multitrajet, avant de présenter l'algorithme amélioré.

\section{Modèle de la transmission}

\subsection{Modèle du canal}

On considère un canal en lien descendant composé de $L$ trajets, de R.I. équivalente en bande de base:

$$
h(\tau, t)=\sum_{l=0}^{L-1} \alpha_{l}(t) \delta\left(\tau-\tau_{l}\right)
$$

où les retards $\tau_{l}$ sont ordonnés en ordre croissant pour $l=0, \ldots, L-1$. Les amplitudes complexes sont notées $\alpha_{l}(t)=\rho_{l}(t) \cdot e^{j \theta_{l}(t)}$. Le signal reçu en bande de base est : 


$$
r(t)=(h * x)(t)+n(t)
$$

où $n(t)$ est un bruit blanc additif Gaussien, complexe. On suppose les retards tels que $\Delta \tau<<T_{s}$, typiquement contenus dans $1 / 4$ de $T_{s}$ par exemple. Le canal étant à bande limitée $W$, on peut considérer un modèle dont l'espacement entre les trajets n'est pas négligeable relativement à $1 / W$. De plus on suppose les amplitudes complexes quasiment invariantes sur 2 symboles voisins.

\subsection{Construction du signal émis}

\subsubsection{Modèle général (modulation linéaire)}

On suppose que le débit global à émettre est partagé en $K$ sous-débits égaux, et que la bande passante disponible est de $W \mathrm{~Hz}$. L'équivalent complexe en bande de base du signal émis (multiplex global) est modélisé par :

$$
x(t)=\sum_{k=0}^{K-1} x_{k}(t)
$$

où

$$
x_{k}(t)=T_{s} \sum_{m=0}^{+\infty} a_{k[m]} \cdot \phi_{k}\left(t-m T_{s}\right)
$$

avec :

- $\phi_{k}(\tau)$ : la forme d'onde d'émission associée à la voie (sous débit) numéro $k$,

- $a_{k[m]}:$ les symboles QPSK de la voie \# $k$, transmis aux instants " $m T_{s}$ ", où $\mathrm{m} \in Z$, et $T_{s}$ est le Temps symbole. Les symboles sont décorrélés, centrés, et de variance $A^{2}$.

\subsubsection{Cas du CDMA}

En CDMA (Coded Division Multiple Access), chaque forme d'onde $\phi_{k}(\tau)$ est construite à partir d'un code qui lui est propre, et utilise toute la bande $W$. Les $Q$ éléments binaires («chips», éventuellement complexes) $\left\{c_{k[q]}, q=0 \ldots Q-1\right\}$ du code \# $k$, sont distribués sur toute la durée symbole, et mis en forme par un filtre $1 / 2$ Nyquist ( $\left.h_{e}(\tau)\right)$ en racine de cosinus surélevé :

$$
\phi_{k}(\tau)=\sum_{q=0}^{Q-1} c_{k[q]} \cdot h_{e}\left(\tau-q T_{c}\right)
$$

où :

- $T_{c}=T_{s} / Q$ désigne le temps chip, qui est donc de l'ordre de $1 / W$ secondes. Plus précisément, $W=\left(1+r_{o f f}\right) / T_{c}$, où $r_{o f f}$ désigne l'excès de bande de $h_{e}(\tau)$.

- Le nombre de sous-débits, $K$, est inférieur ou égal au facteur d'étalement, $Q$.

La technique CDMA étant une technique d'accès multiple, les sous-débits peuvent appartenir à différents utilisateurs.

On suppose les $K$ codes orthogonaux, ce qui se traduit par $\underline{\underline{C}}^{H} \cdot \underline{\underline{C}}=\underline{\underline{I}}_{K}$, où $\underline{\underline{I}}_{K}$ est la matrice identité (taille $K \times K$ ), $\underline{\underline{C}}$ est la matrice des codes (taille $Q \times K),(.)^{H}$ désigne le transposé Hermitien pour une matrice (ou le filtre adapté pour une fonction $\left(x^{H}(\tau)=x^{*}(-\tau)\right)$. Il en résulte que les
$K$ formes d'ondes sont orthogonales :

$$
T_{s} .<\phi_{k}(\tau), \phi_{k^{\prime}}(\tau)>=\delta_{k, k^{\prime}}
$$

où par définition: $\delta_{k, k^{\prime}}=1$ si $k=k^{\prime}, 0$ sinon,

et $\left\langle\phi_{k}(\tau), \phi_{k^{\prime}}(\tau)\right\rangle \stackrel{\Delta}{=} \int_{-\infty}^{+\infty} \phi_{k}(\tau) . \phi_{k^{\prime}}^{*}(\tau) d \tau$. L'orthogonalité peut aussi s'exprimer en fréquence [7].

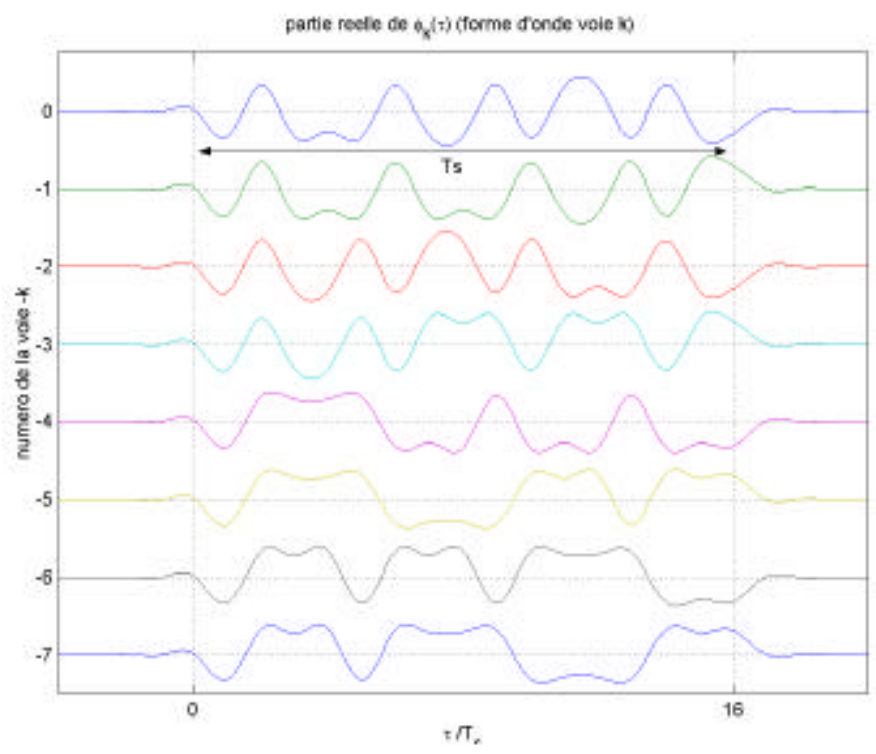

Fig 1. Formes d'ondes $\phi_{k}(\tau)$ en CDMA ( $Q=16$ )

Illustration: la figure 1 présente 8 des 16 formes d'ondes orthogonales, $\phi_{k}(\tau)$, construites à partir de jeux de codes orthogonaux de longueur $Q=16$. Les codes sont construit à partir des séquences orthogonales de Walsh-Hadamard, pondérées par une séquence d'embrouillage unique $[-1+1-1-1-1+1-1-1+1-1+1+1-1+1-1-1]$, issue de la norme UMTS et qui permet d'améliorer les propriétés d'auto-corrélation des séquences initiales. Notons que le code \#0 correspond à la séquence d'embrouillage. L'illustration est réalisée avec des codes réels, mais on a généralement intérêt à rendre les codes complexes. L'excès de bande du filtre $1 / 2$ Nyquist est $r_{\text {off }}=022$.

\subsubsection{Cas de l'OFDM}

La technique OFDM (Orthogonal Frequency Division Multiplexing) utilise un multiplexage des sous-débits à l'aide de $N$ sous-porteuses complexes orthogonales [2], réparties dans la bande $W$, et espacées de $\Delta f=W / N$. Le nombre de voies (ou sous-débits), $K$, est inférieur ou égal au nombre de sous-porteuses, $N$. On supposera par défaut $K=N$. L'obtention d'un "symbole OFDM' à partir des $K$ symboles de l'ensemble des voies est généralement présentée (et réalisée) au moyen d'une Transformée de Fourier Discrète Inverse (TFDI) de taille $N$, suivie du rajout d'un préfixe cyclique (PC), puis d'une mise en forme analogique (Cf partie émission de la figure 6). Nous préfèrerons par défaut la représentation par forme d'onde introduite en (3), avec alors la forme d'onde associée au sous-débit \# $k$ donnée par :

$$
\phi_{k}(\tau)=\exp \left(j 2 \pi f_{k} \tau\right) \cdot \frac{\sqrt{\frac{N+v}{N}}}{T_{s}} \operatorname{Rect}_{\left[-T_{g},+T_{u}\right]}(\tau)
$$


avec:

$$
\begin{aligned}
f_{k} & =k \cdot \frac{W}{N}, \quad \text { pour } k=0, \ldots, \frac{N}{2}-1 \\
& =(k-N) \cdot \frac{W}{N}, \text { pour } k=\frac{N}{2}, \ldots, N-1
\end{aligned}
$$

$$
\text { et : } \quad T_{s}=T_{u}+T_{g} \quad \text { et } \quad T_{u}=N \cdot \frac{1}{W}
$$

où :

- $\operatorname{Rect}_{\left[-T_{g}, T_{u}\right]}(\tau)$ est une fonction rectangulaire ${ }^{1}$ égale à 1 pour $\tau$ compris entre $-T_{g}$ et $+T_{u}$, et nulle ailleurs,

- $T_{g}$ désigne le temps de garde, et $T_{u}$ la partie "utile" du temps symbole.

Le début (préfixe cyclique de durée $T_{g}$ ) de la forme d'onde correspond à une recopie de la fin de la forme d'onde:

$$
\phi_{k}(\tau)=\phi_{k}\left(\tau+T_{u}\right), \text { pour } \tau \in\left[-T_{g}, 0[\right.
$$

La forme d'onde peut ainsi se déduire d'une forme initiale plus courte (durée $\left.T_{u}\right) \psi_{k}(\tau)$, telle que :

$$
\psi_{k}(\tau)=\left\{\begin{array}{c}
\phi_{k}(\tau), \text { pour } \tau \in\left[0,+T_{u}[\right. \\
0 \text { sinon }
\end{array}\right.
$$

Les $K$ formes d'onde initiales (sans préfixe) sont orthogonales. Les familles de formes d'onde initiales et avec préfixe sont biorthogonales: $T_{s} .<\phi_{k}(\tau), \psi_{k^{\prime}}(\tau)>=\delta_{k, k^{\prime}}$

Note Annexe: Représentation à partir des échantillons issus de la Transformée de Fourier Discrète Inverse: En se plaçant en limite du théorème d'échantillonnage, le signal OFDM peut être décrit de manière équivalente à temps discret, au pas $T_{c}=1 / W=T_{u} / N$. Les $N$ échantillons $x_{[m, q]}$ du symbole OFDM numéro $m$, correspondant à $x(t)$ aux instants $q T_{c}+m T_{s}$ pour $q=0 \ldots N-1$, peuvent s'obtenir au moyen d'une TFD inverse de taille $N$ appliquée à l'ensemble des symboles de chacune des $K$ voies (complétés jusqu'à $N$ par des zéros si nécessaire) :

soit:

$$
x_{[m, q]}=\sum_{k=0}^{K-1} a_{k[m]} . \exp \left(j 2 \pi \frac{k q}{N}\right), \quad q=0 \ldots N-1 .
$$

avec

$$
\underline{x}_{[m]}=T F D I_{N}\left\{\underline{a}_{[m]}\right\}
$$

$\underline{a}_{[m]}=\left[a_{0[m]}, \ldots, a_{K-1[m]}, 0, \ldots 0\right]^{T}, \underline{x}_{[m]}=\left[x_{[m, 0]}, \ldots, x_{[m, N-1]}\right]^{T}$. Cette propriété est un atout de réalisation important pour l'OFDM. Les $N$ échantillons "utiles" sont ensuite complétés par les $v$ échantillons (indexés de $-v$ à -1 ) du "préfixe cyclique", avec $v=T_{g} . W$, pour former les $v+N=T_{s} . W$ échantillons du symbole OFDM complet. On peut donc, pour chaque voie \#k, construire les formes d'ondes à partir d'un ensemble de $N$ échantillons $\left\{\psi_{k[q]}, q=0 \ldots N-1\right\}$, de manière similaire au CDMA (4). La forme d'onde (de base) s'exprime ainsi directement par interpolation des échantillons $\psi_{k[q]}=\exp \left(j 2 \pi \frac{k q}{N}\right)$ pour q= $0, \ldots, \mathrm{N}-1$ à l'aide d'un filtre $1 / 2$ Nyquist d'excès de bande nul, $h_{e 0}(\tau)$, soit : $\psi_{k}(\tau)=\sum_{q=0}^{N-1} \psi_{k[q]} \cdot h_{e 0}\left(\tau-q T_{c}\right)$.

${ }^{1}$ En raison de l'utilisation de la fonction rectangulaire, la largeur de bande $W$ n' englobe en réalité que la majeure partie de l'énergie.

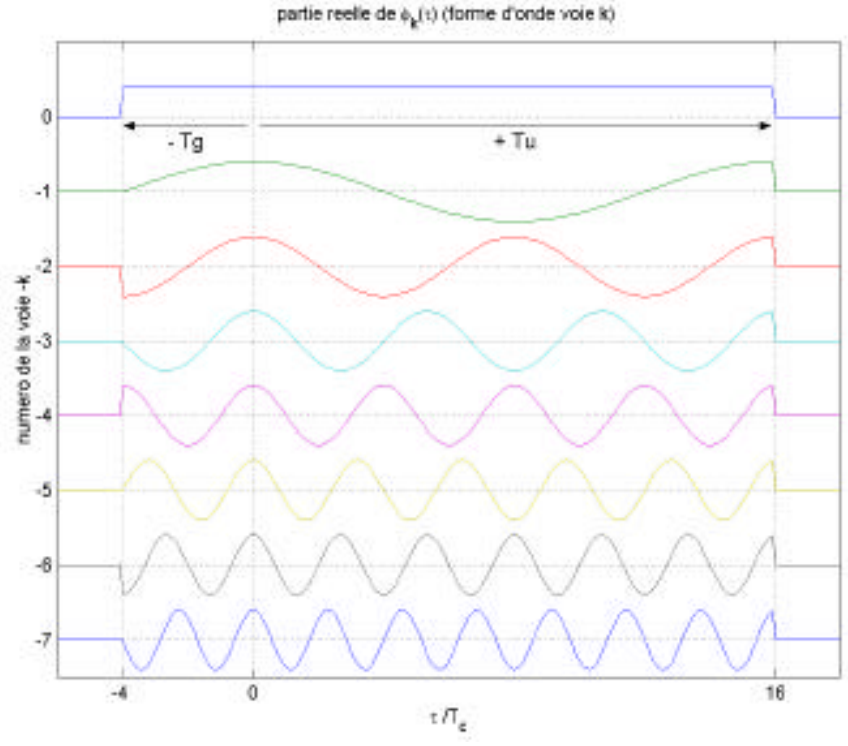

Fig 2. Formes d'ondes $\phi_{k}(\tau)$ en $\operatorname{OFDM}(\mathrm{N}=16, v=4)$

Illustration : la figure 2 présente les parties réelles des 8 premières des $N=16$ formes d'ondes (sous-porteuses orthogonales), et un temps de garde tel que $\mathrm{v}=T_{g} . W=4$.

\section{Boucle à remodulation standard pour la synchronisation de phase à l'aide des décisions}

Une boucle de synchronisation de phase est chargée de construire un estimateur de phase $\hat{\theta}$, utilisé pour corriger la phase des symboles estimés avant la prise de décision. L'objectif de la section est d'abord de présenter la structure et l'algorithme d'estimation que nous considérons, puis d'étudier leur adéquation dans un contexte mono-trajet multi-voie, en particulier en CDMA et en OFDM.

\subsection{Structure et algorithme standard}

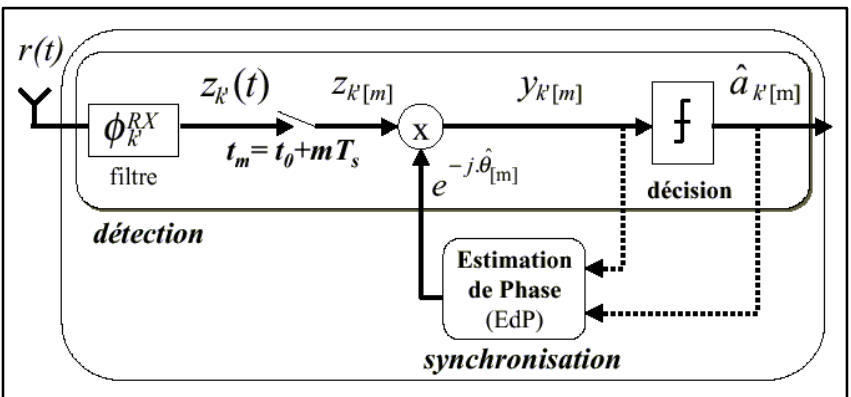

Fig. 3 : Structure équivalente du récepteur avec détection des symboles (voie \# $k^{\prime}$ ) et synchronisation conjointes

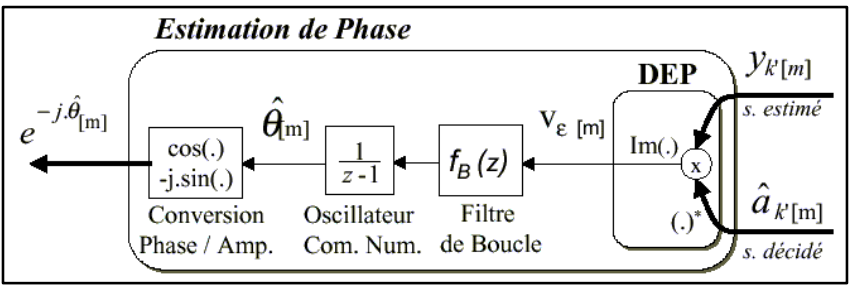

Fig. 4 : Estimation de Phase pour la boucle à remodulation 


\subsubsection{Structure générale du récepteur}

On se place dans la situation où l'estimée de la phase servant pour la correction, $\hat{\theta}$, est obtenue à partir des décisions, mais seulement des symboles de la voie $\# k^{\prime}$, notés $\hat{a}_{k^{\prime}[m]}$. La décision et la synchronisation de phase sont réalisées conjointement (Cf figure 3), après les opérations (ou opérations équivalentes) de:

- $\quad$ filtrage de réception : $z_{k^{\prime}}(t)=\left(\phi_{k^{\prime}}^{R X} * r\right)(t)$ où $\phi_{k^{\prime}}^{R X}(\tau)$ est la R.I. du filtre de réception,

- échantillonnage synchrone aux instants symboles $t_{m}=t_{0}+m T_{s}: \quad z_{k^{\prime}[m]}=z_{k^{\prime}}\left(t_{0}+m T_{s}\right)$,

où le délai d'échantillonnage $t_{0}$ est paramétrable en fonction du (des) retard du canal,

- et de correction de phase à l'aide de l'estimé $\hat{\boldsymbol{\theta}}_{[m]}$ à l'instant $t_{m}: y_{k\lceil m]}=z_{k^{\prime}[m]} \cdot e^{-j \hat{\theta_{[m]}}}$,

pour alimenter (exclusivement ou non) l'organe de décision, et celui de synchronisation.

A partir de cette structure générale de synchronisation / détection, l'algorithme (donné en (9) et (10)) d'Estimation de Phase $(E d P)$ doit permettre de réduire la rotation de phase affectant les symboles avant décision.

\subsubsection{Structure de la boucle de synchronisation}

On considère la structure générale d'une boucle à verrouillage de phase numérique (Cf figure 4): l'estimation de phase est mise à jour de manière itérative au rythme symbole, à l'aide d'un signal d'erreur de phase $v_{\varepsilon}$, filtré par un filtre de boucle $\left(f_{B}\right)$, selon :

$$
\hat{\theta_{[m+1]}}=\hat{\theta_{[m]}}+\left(f_{B} * v_{\varepsilon}\right)_{[m]}
$$

\subsubsection{Détecteur d'erreur de phase standard}

Le détecteur d'erreur de phase (DEP) calcule le signal d'erreur, à partir du signal filtré $z_{k^{\prime}}(t)=\left(r * \phi_{k^{\prime}}^{R X}\right)(t)$, corrigé en phase, et pondéré (ou remodulé) par le symbole décidé $\hat{a}_{k^{\prime}[m]}$ (ou connu en cas d'une séquence pilote) :

$$
v_{\varepsilon[m]}=\operatorname{Im}\left\{e^{-j \hat{\theta}_{[m]}} \cdot \hat{a}_{k^{\prime}[m]}^{*} \cdot z_{k^{\prime}}\left(m T_{s}+t_{0}\right)\right\}
$$

L'estimation de phase est donc basée ici sur une rétroaction ("feed-back") [1], puisque le signal d'erreur est construit à partir de $\hat{\theta}_{[m]}$, comme illustré sur la figure 3 . Avec les notations introduites, le signal d'erreur consiste simplement en $v_{\varepsilon[m]}=\operatorname{Im}\left\{\hat{a}_{k^{\prime}[m]}^{*} \cdot y_{k^{\uparrow}[m]}\right\}$.

Un tel signal d'erreur compense la modulation des symboles (en supposant les décisions justes), et ramène (en l'absence d'interférence) à une caractéristique sinusoïdale pour le détecteur de phase.

Note annexe: Cet algorithme standard (et structure de réception associée) trouve son origine dans l'estimation par Maximum de Vraisemblance de la phase (désirée) $\hat{\theta_{0}}$ d'un canal mono-trajet, dans un contexte plus restrictif, et avec comme filtrage de réception, le filtre adapté à la forme d'onde d'émission de la voie \# $k^{\prime}$ unique (Cf [1], [8]).

\section{A propos de l'évaluation des performances:}

Etant donné la boucle à remodulation imposée, on va s'intéresser dans la suite à son comportement dans un contexte de canal mono-trajet puis multi-trajet, CDMA ou OFDM. En particulier, on devra déterminer pour des phases d'entrée fixes du canal, quel est le point d'équilibre stable de la boucle, $\hat{\boldsymbol{\theta}}_{e q}$, et si il correspond bien à la phase que l'on souhaitait compenser (à préciser selon le contexte), nommée phase désirée, $\theta_{\text {des }}$. On évaluera l'erreur $\varepsilon$ entre la phase désirée et la phase estimée. Les performances d'estimation (biais, variance) d'une boucle de phase se déduisent de manière générale par une analyse classique du signal d'erreur $v_{\varepsilon}$ basée sur la S-courbe et le bruit de boucle, comme rappelé en annexe A.1.

\subsection{Comportement en contexte multi-voie mono-trajet}

On suppose d'abord un canal mono-trajet de paramètre d'amplitude $\rho_{0}=1$, de retard $\tau_{0}$ parfaitement estimé, et de phase $\theta_{0}(t)$. Le signal reçu en bande de base est:

$$
r(t)=\exp \left(j \theta_{0}(t)\right) \cdot x\left(t-\tau_{0}\right)+n(t)
$$

\subsubsection{Caractérisation générale}

La boucle à remodulation étant initialement prévue pour un contexte mono-utilisateur, voyons comment évolue la qualité du signal d'erreur en contexte multi-voie. Pour cela, il suffit d'après (10) de vérifier si la sortie du filtre de réception correctement échantillonnée, $z_{k^{\prime}[m]}$, présente de bonnes caractéristiques pour l'estimation de phase.

Avant échantillonnage, le signal en sortie du filtre de réception est:

$z_{k^{\prime}}(t)=T_{s} \sum_{k=0}^{K-1} \sum_{m} a_{k[m]} \cdot \beta_{k k^{\prime}}\left(t-m T s-\tau_{0}\right) \cdot e^{j \theta_{0}(t)}+\eta_{k^{\prime}}(t)$

où :

- $\eta_{k^{\prime}}(t)$ est le bruit filtré, égal à $\left(\phi_{k^{\prime}}^{R X} * n\right)(t)$,

- $\beta_{k k^{\prime}}(t)$ est la forme d'onde émission-réception (E/R) de la voie $k$ vers la voie $k^{\prime}$, définie par :

$$
\beta_{k k^{\prime}}(\tau)=\left(\phi_{k} * \phi_{k^{\prime}}^{R X}\right)(\tau)
$$

Les fonctions $\beta_{k k^{\prime}}(t)$ matérialisent la mise en forme des symboles jusqu'à l'échantillonneur, sans l'effet du canal. Elles sont propres à l'équipement $\mathrm{E} / \mathrm{R}$, et ainsi au type de modulation utilisée. Par convention, nous utilisons une représentation non causale du filtre de réception $\phi_{k^{\prime}}^{R X}(t)$, de sorte que $\beta_{k k^{\prime}}(t)$ ait son maximum (réel) en zéro.

Nous nous placerons toujours dans le cas (le plus intéressant) où la forme d'onde des équipements $\mathrm{E} / \mathrm{R}$ est construite en respectant le critère de Nyquist multi-voie:

$$
\beta_{k k^{\prime}}\left(n T_{s}\right)=\beta_{k^{\prime} k^{\prime}}(0) . \delta_{k^{\prime} k[n]}
$$

équivalent à (15) et (16) :

$$
\left\{\begin{array}{l}
\beta_{k k^{\prime}}\left(n T_{s}\right)=\beta_{k k^{\prime}}(0) \cdot \delta_{[n]}, \quad \forall n \in Z \\
\beta_{k k^{\prime}}(0)=\beta_{k^{\prime} k^{\prime}}(0) \cdot \delta_{k k^{\prime}}, \forall k=0 \ldots K-1
\end{array}\right.
$$


En choisissant le délai d'échantillonnage égal au retard de propagation (supposé parfaitement estimé), $t_{0}=\tau_{0}$, le critère (14) assure l'absence d'Interférence Entre Symboles (IES) ainsi que l'Interférence Entre Voies (IEV). Le signal en sortie du filtre de réception ainsi échantillonnée, $z_{k^{[}[\mathrm{m}]}$, ne dépend en effet que du symbole désiré :

$$
z_{k^{\prime}[m]}=\beta_{k^{\prime} k^{\prime}[0]} \cdot e^{+j \theta_{0[m]}} \cdot a_{k^{\prime}[m]}+\eta_{k^{\prime}[m]}
$$

où le coefficient réel de pondération $\beta_{k^{\prime} k^{\prime}[0]}=T_{s} \cdot \beta_{k^{\prime} k^{\prime}}(0)$ représente l'échantillonnée au retard nul de la forme d'onde E/R analogique de la voie désirée, $\beta_{k^{\prime} k^{\prime}}(t)$.

Le déphasage à corriger (car affectant le symbole désiré), correspond alors au déphasage du canal de propagation: $\theta_{d e s}=\theta_{0}$. L'erreur résiduelle entre la phase estimée, $\hat{\theta}_{0[m]}$, et la vraie phase du trajet, $\theta_{0[m]}=\theta_{0}\left(t_{m}\right)$, à l'instant $t_{m}$ est:

$$
\varepsilon_{[m]}=\theta_{0[m]}-\hat{\theta}_{o[m]}
$$

Le signal d'erreur $v_{\varepsilon[m]}=\operatorname{Im}\left\{\hat{a}_{k^{\prime}[m]}^{*} \cdot e^{-j \hat{\theta}_{0[m]}} \cdot z_{k^{\prime}[m]}\right\}$ se résume (en supposant toujours $\hat{a}_{k \uparrow m]}=a_{k \uparrow m]}$ ) à:

$$
v_{\varepsilon[m]}=\underbrace{A^{2} \cdot \beta_{k^{\prime} k^{\prime}[0]} \cdot \sin \left(\varepsilon_{[m]}\right)}_{\text {utile }}+\underbrace{\operatorname{Im}\left\{\eta_{k^{\prime}[m]} \cdot \hat{a}_{k^{\prime}[m]}^{*} \cdot e^{-j \hat{\theta}_{0[m]}}\right\}}_{\text {bruit }}
$$

Grâce à l'absence d'interférence, la composition du signal d'erreur apparaît identique à celle que l'on obtiendrait en contexte mono-voie (mono-utilisateur), ce qui assure les mêmes bonnes performances. On retrouve donc une caractéristique de détecteur de phase avec une forme classique sinusoïdale, que l'on identifie par la SCourbe (Cf annexe A.1), obtenue en n'observant que la partie déterministe du signal d'erreur (en réponse à une valeur constante donnée de l'erreur de phase):

$$
S_{P}(\varepsilon) \stackrel{\Delta}{=} E\left\{v_{\varepsilon[m]} \mid \varepsilon\right\}=A^{2} \cdot \beta_{k^{\prime} k^{\prime}[0]} \cdot \sin (\varepsilon)
$$

Notons qu'avec des formes d'ondes E/R sans IES ((15) respectée) mais avec IEV ((16) non respectée), on obtiendrait une S-courbe identique (estimation sans biais), mais la partie aléatoire du signal d'erreur aurait une variance plus élevée due aux termes d'interférence.

\subsubsection{Application en CDMA}

Pour le récepteur conventionnel en CDMA, le filtre de réception est le filtre adapté à la forme d'onde d'émission sur la voie "désirée", $\phi_{k^{\prime}}^{H}(\tau)=\phi_{k^{\prime}}^{*}(-\tau)$. Dans ce cas, la forme d'onde $E / R \quad \beta_{k k^{\prime}}(\tau)$ n'est autre que la fonction d'inter-corrélation à durée finie $\gamma_{k k^{\prime}}(\tau)=\left(\phi_{k} * \phi_{k^{\prime}}^{H}\right)(\tau)$ entre les formes d'ondes des voies $k$ et $k^{\prime}$ :

$$
\phi_{k^{\prime}}^{R X}(\tau)=\phi_{k^{\prime}}^{H}(\tau) \quad \text { et } \quad \beta_{k k^{\prime}}(\tau)=\gamma_{k k^{\prime}}(\tau)
$$

Le critère (14) est bien vérifié puisque les codes sont orthogonaux, et qu'ils sont mis en forme par des filtres $1 / 2$ Nyquist. Cependant, à la moindre erreur d'échantillonnage (sur le choix de $t_{0}$ lors de la numérisation de $z_{k^{\prime}}(t)$ ), de l'interférence entre symboles et entre voies apparaîtrait ((15) et (16) en échec). En CDMA, l'interférence IEV est appelée Interférence d'Accès Multiple (IAM).

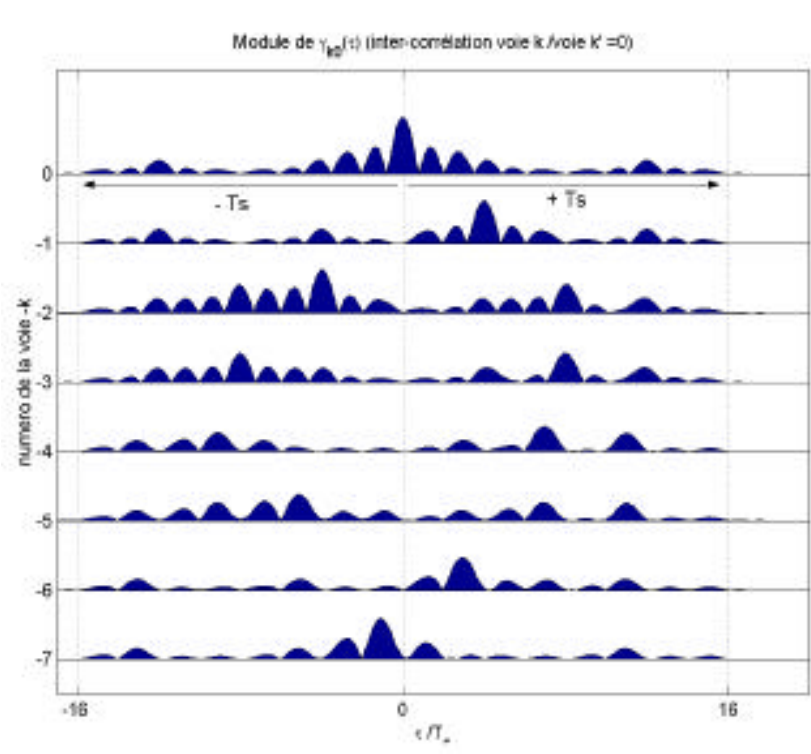

Fig. 5 : Exemple de formes d'ondes $E / R \beta_{k k^{\prime}}(\tau)=\gamma_{k k^{\prime}}(\tau)$ (inter-corrélations) en CDMA

Illustration : la figure 5 présente les modules de $8(k=0$ ...7) des 16 formes d'ondes "émission-réception", $\beta_{k k^{\prime}}(\tau)=\gamma_{k k^{\prime}}(\tau)$, relatives à la voie désirée $\# k^{\prime}=0$, obtenues à partir des formes d'onde de la figure 1. On remarque d'une part sur la voie désirée l'étroitesse (largeur de l'ordre de 2. $\frac{1}{W} \approx 2 . T_{c}$ ) du pic principal d'autocorrélation, $\gamma_{k^{\prime} k^{\prime}}(\tau)$, grâce à la large-bande (de l'ordre de $W$ ) de la forme d'onde $\phi_{k^{\prime}}(\tau)$. On vérifie aussi que les formes d'ondes E/R interférentes $(k \neq k$ ') sont nulles au retard nul (orthogonalité), et que les fonctions sont toutes nulles aux retards symboles suivant ou précédant (absence d'IES). Par ailleurs ces deux propriétés sont brisées au moindre décalage de $\delta t$ (intervention de $\gamma_{k k^{\prime}}\left(n T_{s}+\delta t\right)$ au lieu de $\gamma_{k k^{\prime}}\left(n T_{s}\right)$ ), qui pourra survenir en cas d'erreur d'échantillonnage ou en présence d'un trajet secondaire de délai non nul $\tau_{1}-\tau_{0}$ par rapport au trajet principal.

\subsubsection{Application en OFDM}

En OFDM, on présente généralement les opérations du récepteur conventionnel comme étant la suppression de l'intervalle de garde dans le signal reçu (ce qui nécessite le positionnement de la fenêtre "utile", d'un symbole OFDM) suivie d'une TFD pour restituer l'ensemble des symboles des $\mathrm{K}$ différentes voies. En utilisant la représentation par formes d'onde, ces opérations sont bien équivalentes (en ne s'intéressant qu'aux symboles de la voie $\# k^{\prime}$ ) à celles du récepteur linéaire présenté en sections 3.1 et 3.2.1. Le filtre de réception utilisé dans le récepteur conventionnel n'est alors pas le filtre adapté à la forme d'onde complète d'émission, $\phi_{k^{\prime}}^{H}(\tau)$ (pourtant optimal dans le cas monotrajet) mais le filtre adapté à la forme d'onde de base, sans le préfixe cyclique (filtre adapté tronqué ):

$$
\phi_{k^{\prime}}^{R X}(\tau)=\psi_{k^{\prime}}^{H}(\tau) \quad \text { et } \quad \beta_{k k^{\prime}}(\tau)=\left(\phi_{k} * \psi_{k^{\prime}}^{H}\right)(\tau)
$$

Le choix de la portion de signal en entrée de la TFD est équivalent au choix de $t_{0}$ dans notre représentation. 


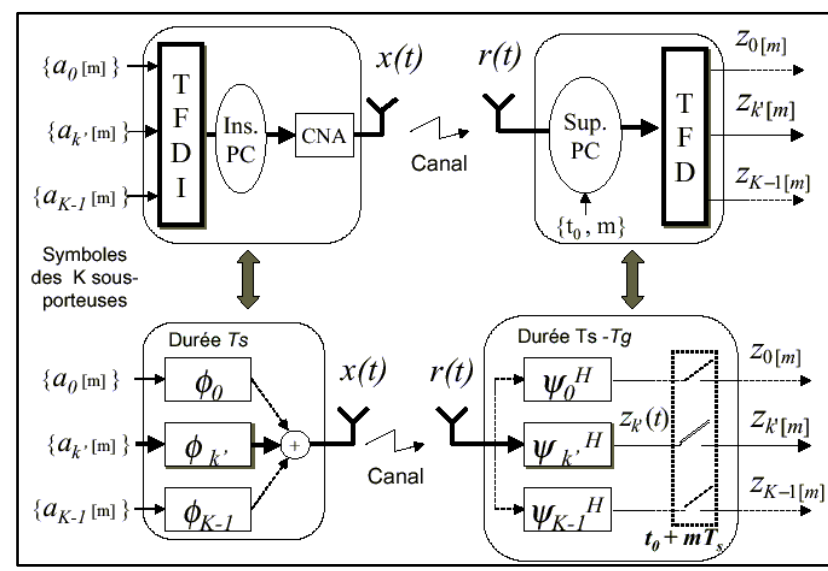

Fig. 6 : Equivalence des représentations d'une chaîne OFDM

Compte tenu des supports des fonctions $\phi_{k}$ et $\psi_{k^{\prime}}$, la forme d'onde $E / R \quad \beta_{k k^{\prime}}(\tau)$ a un support non-nul s'étendant de $-T_{s}=-\left(T_{u}+T_{g}\right)$ à seulement $+T_{u}$, ce qui laisse une marge égale au temps de garde, $T_{g}$ pour l'absence d'IES ((15) vérifiée). L'utilisation du préfixe cyclique amène une propriété encore plus intéressante, c'est le maintien à zéro des formes d'ondes $\mathrm{E} / \mathrm{R}$ interférentes $(k \neq k$ ') non pas seulement au retard nul ((16) vérifiée), mais sur une plage de retard égale au temps de garde, soit sur $\left.]-T_{g}, 0\right]$. Cette propriété est illustrée par la figure 7 et justifiée dans l'équation (20). Globalement, il y aura donc absence totale d'interférence non seulement pour un échantillonnage idéal $\left(t_{0}=\tau_{0}\right)$, mais aussi en cas de décalage vers la gauche tel que $\left.\left.t_{0}=\tau_{0}-\delta \in\right] \tau_{0}-T_{g} ; \tau_{0}\right] \quad$ (coefficients $\beta_{k k^{\prime}}(n T s-\delta)$ intervenant dans le calcul de $y_{k^{\prime}}\left(m T s+\tau_{0}-\delta\right)$ selon (12), sont nuls pour $k \neq k^{\prime}$ ou $n \neq 0$ ).

On voit donc que dans la technique OFDM (avec préfixe cyclique): le sacrifice d'une portion de symbole à la réception permet de maintenir l'orthogonalité des formes d'ondes (également l'absence d'IES) sur une plage de retard égale au temps de garde, ce qui sera utile en cas d'erreur sur l'estimation de retard ou en présence de trajets multiples pour éviter l'IEV, appelée Interférence Entre Sous-Porteuses (IESP) en OFDM.

Calcul annexe : expression de $\beta_{k k^{\prime}}(\tau)$ en OFDM La forme d'onde $\mathrm{E} / \mathrm{R}$ peut s'évaluer par :

$$
\begin{aligned}
\beta_{k k^{\prime}}(\tau) & =\int_{-T g}^{+T_{u}} \phi_{k}(u) \cdot \psi_{k^{\prime}}^{*}(u-\tau) d u \\
& =\int_{0}^{T_{u}} \psi_{k^{\prime}}^{*}(u) \cdot \phi_{k}(u+\tau) d u
\end{aligned}
$$

- Pour les $\tau>0, \beta_{k k^{\prime}}(\tau)$ coïncide avec la fonction d'intercorrélation à durée finie entre les formes de base $\psi_{k}$ et $\psi_{k^{\prime}}$, soit $\left(\psi_{k} * \psi_{k^{\prime}}^{H}\right)(\tau)=\frac{\frac{N+v}{N}}{T_{s}^{2}} e^{j 2 \pi f_{k} \tau} \int_{0}^{T_{u}-\tau} e^{j 2 \pi\left(f_{k}-f_{k}\right) u} d u$.

- Alors que pour $\left.\tau \in]-T_{g} ; 0\right], \beta_{k k^{\prime}}(\tau)$ correspond à la fonction d'intercorrélation périodique entre $\psi_{k}$ et $\psi_{k^{\prime}}$, soit $\frac{\frac{N+v}{N}}{T_{s}^{2}} e^{j 2 \pi f_{k} \tau} \int_{0}^{T_{u}} e^{j 2 \pi\left(f_{k}-f_{k^{\prime}}\right) u} d u$, qui est nulle lorsque $k \neq k^{\prime}$, et de module constant si $k=k^{\prime}$ :

$$
\text { si } \left.\tau \in]-T_{g} ; 0\right]: T_{s} \beta_{k k^{\prime}}(\tau)=\left\{\begin{array}{cr}
e^{j 2 \pi f_{k^{\prime}} \tau} & \text { si } k=k^{\prime} \\
0 & \text { si } k \neq k^{\prime}
\end{array}\right.
$$

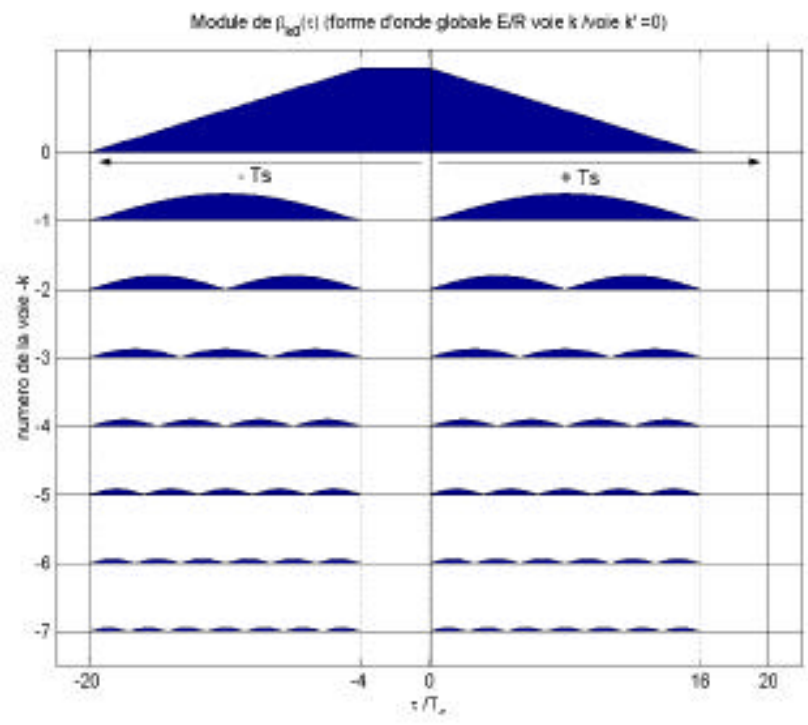

Fig. 7 : Exemple de formes d'ondes émission-réception $\beta_{k k^{\prime}}(\tau)$ en $\operatorname{OFDM}(N=16, v=4)$

Illustration : la figure 7 présente les modules de $8(k=0$ ...7) des 16 formes d'ondes "émission-réception", $\beta_{k k^{\prime}}(\tau)$, relatives à la voie désirée \# $k^{\prime}=0$, obtenues à partir des formes d'onde de la figure 2. Elles mettent en évidence, comparativement aux courbes obtenues en CDMA (figure 5), d'une part la marge sur la plage d'échantillonnage, et d'autre part la largeur des pics $\gamma_{k^{\prime} k^{\prime}}(\tau)$ obtenus sur la voie désirée (largeur de l'ordre de $\left(2 T_{s}-T_{g}\right)$, avec un palier maximum constant sur une durée $T_{g}$ ), dû au fait que les formes d'ondes $\phi_{k^{\prime}}(\tau)$ sont à bande-étroite (de l'ordre de $\Delta f=W / N)$.

\section{Notes:}

1) si l'échantillonnage était en avance de $\delta$ par rapport au délai de propagation, soit $\left.\left.t_{0}=\tau_{0}-\delta \in\right] \tau_{0}-T_{g} ; \tau_{0}\right]$, le déphasage $\theta_{\text {des }}$ qu'estimerait sans biais la boucle serait $\theta_{d e\{m]}=\theta_{0[m]}-2 \pi f_{k^{\prime}} \delta$. On aurait en effet un déphasage supplémentaire (introduit par le coefficient $T_{s} \beta_{k^{\prime} k^{\prime}}(-\delta)$ $\left.=\exp \left\{-j\left(2 \pi f_{k^{\prime}} \delta\right)\right\}\right)$ variant linéairement avec le numéro $k^{\prime}$ de la sous-porteuse. Ainsi, si l'on dispose de plusieurs voies $\# k^{\prime}$ (plusieurs sous-porteuses pilotes), on pourra à partir de l'ensemble des phases $\hat{\theta}_{\left\{k^{\prime}\right\}}$ estimées sur chaque voie $k^{\prime}$ retrouver conjointement la phase du trajet, $\theta_{0}$, et l'avance d'échantillonnage, $\delta$.

2) l'algorithme mono-voie standard considéré ici estime la phase du canal de propagation en n'exploitant qu'une seule voie de symboles, $\# k^{\prime}$, après démultiplexage (i.e. après TFD). Rappelons tout de même qu'en OFDM, une classe importante d'algorithmes d'estimation (du retard, du décalage de fréquence, ...) travaille directement à partir du signal multi-voie complet reçu, $r(t)$, en exploitant la redondance introduite par le préfixe cyclique [4]. Par exemple, l'argument de la fonction de corrélation entre les échantillons du préfixe cyclique et ceux de la fin du symbole traduit l'incrément de phase, du moins tant que le canal est mono-trajet ... 


\section{Comportement en présence de trajets secondaires}

On considère désormais le canal complet avec $L$ trajets (1), de retards $\tau_{l}$ (ordonnés en ordre croissant) connus, et d'amplitudes complexes $\quad \alpha_{l}(t)=\rho_{l}(t) \cdot e^{j \theta_{l}(t)} \quad$ (initialement bien estimés). Le signal reçu en bande de base est :

$$
r(t)=\sum_{l=0}^{L-1} \alpha_{l}(t) x\left(t-\tau_{l}\right)+n(t)
$$

\subsection{Caractéristiques générales}

On suppose le récepteur constitué du même filtre de réception que précédemment (section 3), avec en sortie:

$$
z_{k^{\prime}}(t)=T_{s} \sum_{k=0}^{K-1} \sum_{m} a_{k[m]} \cdot \chi_{k, k^{\prime}}(t-m T s)+\eta_{k^{\prime}}(t)
$$

où de manière générale : $\quad \chi_{k, k^{\prime}}(\tau)=\left(\beta_{k k^{\prime}} * h\right)(\tau)$

et pour un canal à $L$ trajets:

$$
\chi_{k, k^{\prime}}(\tau)=\sum_{l=0}^{L-1} \alpha_{l}(t) \cdot \beta_{k k^{\prime}}\left(\tau-\tau_{l}\right)
$$

$\chi_{k, k^{\prime}}(\tau)$ représente ici la forme d'onde de la chaîne globale "Emission/Canal/Réception", (E/C/R), de la voie \#k vers la voie $\# k^{\prime}$ incluant le canal. Elle résulte de la superposition de $L$ versions retardées, atténuées et déphasées de la forme E/R de l'équipement, $\beta_{k k^{\prime}}(t)$.

Considérons le résultat de l'échantillonnage $z_{k^{\prime}[m]}=z_{k^{\prime}}\left(t_{0}+m T_{s}\right)$ obtenu aux instants $t_{m}=t_{0}+m \cdot T_{s}$, où le choix du décalage $t_{0}$ sera discuté ultérieurement pour les cas spécifiques CDMA et OFDM. On a:

$$
\begin{aligned}
z_{k^{\prime}[m]}= & \underbrace{a_{k^{\prime}[m]} \cdot T_{s} \chi_{k^{\prime} k^{\prime}}\left(t_{0}\right)}_{\text {utile }}+\underbrace{\eta_{k^{\prime}[m]}}_{\text {bruit }} \\
& +\underbrace{T_{s} \sum_{n \neq m} a_{k^{\prime}[n]} \cdot \chi_{k^{\prime} k^{\prime}}\left(t_{0}+(m-n) T_{s}\right)}_{I E S_{k^{\prime}}} \\
& +\underbrace{T_{s} \sum_{n} \underbrace{\sum_{k \neq k^{\prime}} a_{k[n]} \cdot \chi_{k k^{\prime}}\left(t_{0}+(m-n) T_{s}\right)}_{I E V_{k^{\prime}}\{m-n\}}}_{I E V_{k^{\prime}}}
\end{aligned}
$$

L'échantillon $z_{k^{\prime}[m]}$ contient des termes d'interférence provenant de la même voie \# $k^{\prime}$, notés $I E S_{k^{\prime}}$, ou provenant des voies autres que $\# k^{\prime}$, notés $I E V_{k^{\prime}}$. Ces termes apparaissent par l'intermédiaire des coefficients $\chi_{k k^{\prime}[n]}=T_{s} \chi_{k k^{\prime}}\left(t_{0}+n T_{s}\right)$, qui dépendent cette fois du canal, et pas seulement des formes d'onde des équipements $E / R$. Le $1^{o}$ terme de droite dans l'équation (24) est le seul construit à partir du symbole désiré, pondéré par le coefficient $\chi_{k^{\prime} k^{\prime}}\left(t_{0}\right)$ : il représente entièrement la partie "utile", pour la détection, mais il n'en sera pas forcément de même pour la synchronisation, comme nous le verrons en 4.2. En terme de synchronisation, si on utilise une boucle à remodulation dont le signal d'erreur $v_{\varepsilon[m]}$ est construit à partir des échantillons de la voie $\# k^{\prime}$, $z_{k^{\prime}[m]}=z_{k^{\prime}}\left(t_{0}+m T s\right)$, selon l'algorithme standard (10), on peut se demander, conjointement au choix du délai d'échantillonnage $t_{0}$ : Quelle phase adéquate $\theta_{\text {des }}$, en fonction des paramètres du canal, doit on chercher à estimer? Le sens du mot "adéquation"' étant pris au sens d'intérêt pour le récepteur, mais également de possibilité, c'est à dire de qualité de l'estimation $\hat{\theta}$ de $\theta_{\text {des }}$ délivrée par la boucle. Nous allons répondre spécifiquement pour les cas CDMA et OFDM, mais avant cela, établissons l'expression générale du bruit de boucle $\left(N_{P}\right)$, et de la $\mathrm{S}$ courbe, $S_{P}(\varepsilon)$ qui composent le signal d'erreur. Les symboles et le bruit étant décorrélés, les termes de bruit additif et d'interférence $\left(I E S_{k^{\prime}}\right.$ et $\left.I E V_{k^{\prime}}\right)$ ne génèrent que du bruit de boucle, noté (en omettant l'indice [m]) :

$$
N_{P}=\operatorname{Im}\left\{\left(\eta_{k^{\prime}}+I E S_{k^{\prime}}+I E V_{k^{\prime}}\right) \cdot \hat{a}_{k^{\prime}}^{*} \cdot e^{-j \hat{\theta}}\right\}
$$

La S-courbe, qui représente l'espérance de $v_{\varepsilon}$ (Cf (10)) conditionnellement à des valeurs fixes de $\hat{\theta}$, ne provient que du $1^{o}$ terme de droite (partie utile pour la détection) de la formule (24), et est donnée par:

$$
S_{P}=A^{2} T_{s} \cdot \operatorname{Im}\left\{\chi_{k^{\prime} k^{\prime}}\left(t_{0}\right) \cdot e^{-\hat{\theta}}\right\}
$$

La forme de la S-courbe dépend donc uniquement du coefficient $T_{s} \chi_{k^{\prime} k^{\prime}}\left(t_{0}\right)$, qui représente l'échantillonnée de la mise en forme émission-réception convoluée par le canal, et regroupe donc les contributions des différents trajets, selon (23). Le point d'équilibre stable (obtenu pour $S_{P}=0$ ) coïncidera donc avec la phase qui affecte les symboles désirés de la voie $k^{\prime}$ :

$$
\hat{\theta}_{e q}=\operatorname{Arg}\left\{\chi_{k^{\prime} k^{\prime}}\left(t_{0}\right)\right\}
$$

Voyons maintenant ce que l'on peut en déduire pour les cas spécifiques CDMA et OFDM.

\section{2. en CDMA : une boucle par trajets?}

En CDMA, grâce à la large-bande des formes d'ondes d'émission, et à la localisation en temps des fonctions d'auto-corrélations $\gamma_{k^{\prime} k^{\prime}}(\tau)=\beta_{k^{\prime} k^{\prime}}(\tau)$, on peut chercher à poursuivre individuellement les phases de chaque trajet $\theta_{l}$, en faisant une boucle par trajet. L'intérêt est clair pour le récepteur CDMA, qui pourra utiliser la connaissance des phases de chaque trajet pour améliorer la détection.

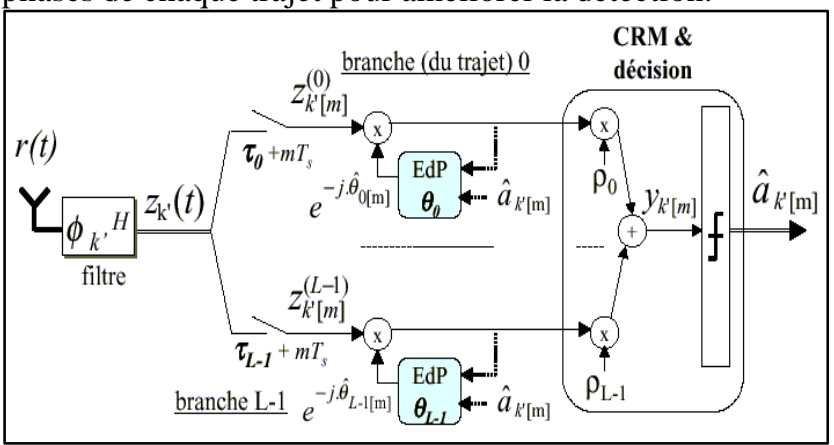

Fig. 8: Schéma (équivalent) de synchronisation des phases des trajets et de détection dans un Récepteur Rake en CDMA

La partie synchronisation du récepteur est formée alors de $L$ boucles de synchronisation construites selon le schéma standard dupliqué (à priori non-optimal pour estimer les $L$ 
phases, mais relativement simple), en réglant le délai d'échantillonnage $t_{0}^{(l)}$ de la boucle numéro \# $l$ sur le délai du trajet $l$, soit : $t_{0}^{(l)}=\tau_{l}$. Sur la branche \# $l$, la variable discrète $z_{k^{\prime}[m]}^{(l)}=z_{k^{\prime}}\left(\tau_{l}+m T_{s}\right)$, obtenue par échantillonnage approprié en sortie du filtre de réception, est utilisée de manière standard pour construire le signal d'erreur $v_{\varepsilon[m]}^{(l)}$.

La partie “détection"' du récepteur doit être revue pour prendre en compte l'aspect multi-trajet. Nous considérons ici par défaut le détecteur "Rake" qui forme la variable de décision $y_{k^{\prime}[m]}$ de la voie $k^{\prime}$ en faisant une Combinaison cohérente à Rapport signal à bruit Maximum (CRM) des échantillons $z_{k \uparrow m]}^{(l)}$ en sortie des $L$ branches, soit:

$$
y_{k^{\prime}[m]}=\sum_{l=0}^{L-1} z_{k^{\prime}}\left(\tau_{l}+m T_{s}\right) \cdot \rho_{l} \cdot \exp \left(-j \hat{\boldsymbol{\theta}}_{l[m]}\right)
$$

On se concentre maintenant sur la boucle de synchronisation du $l^{\prime \text { eme }}$ trajet. Pour réaliser le Rake, il est nécessaire que la boucle estime $\theta_{\text {des }}=\theta_{l^{\prime}}$, en minimisant l'erreur : $\varepsilon_{[m]}^{\left(l^{\prime}\right)}=\theta_{l^{\prime}[m]}-\hat{\theta_{l^{\prime}[m]}}$. Le coefficient $\chi_{k^{\prime} k^{\prime}}\left(t_{0}\right)$ (avec ici $\left.t_{0}=\tau_{l^{\prime}}\right)$, qui intervient dans la S-courbe $S_{p}^{\left(l^{\prime}\right)}\left(\varepsilon_{l}\right)$ ((26 et (23) avec $\beta=\gamma$ ) est donné par :

$$
\chi_{k^{\prime} k^{\prime}}\left(\tau_{l^{\prime}}\right)=\rho_{l^{\prime}} \cdot e^{j \theta_{l^{\prime}}} \cdot \gamma_{k^{\prime} k^{\prime}}(0) .+\sum_{l \neq l^{\prime}} \rho_{l} \cdot e^{j \theta_{l}} \cdot \gamma_{k^{\prime} k^{\prime}}\left(\tau_{l^{\prime}}-\tau_{l}\right)
$$

On voit donc que le déphasage $\theta_{e q}$ (27) affectant le symbole utile, égal à l'argument de $\chi_{k^{\prime} k^{\prime}}\left(\tau_{0}\right)$, correspond bien au déphasage du trajet $l^{\prime}$ lorsque l'approximation habituelle en CDMA, $\gamma_{k^{\prime} k^{\prime}}\left(\tau_{l^{\prime}}-\tau_{l}\right) \approx 0, l \neq l^{\prime}$, est valable. Avec des codes présentant des lobes secondaires d'autocorrélation négligeables par rapport au pic principal, l'approximation est en effet valable du moment que les trajets voisins sont suffisamment espacés vis à vis du temps chip, car alors $\gamma_{k^{\prime} k^{\prime}}\left(\tau_{l^{\prime}}-\tau_{l}\right)$ devient négligeable. Pour l'ensemble des boucles, il faut donc des trajets "résolus", c'est à dire dont les écarts soient au moins de l'ordre de $T_{c}$.

En conclusion, les $L$ boucles de re-modulation standards permettront de bien suivre les phases des trajets dans la mesure où ceux-ci sont résolus et que les codes utilisés ont de bonne propriétés d'auto-corrélation. On aura tout de même une variance d'estimation (obtenue à partir de (25)) augmentée par rapport au cas mono-trajet, en raison de l'interférence (d'accès multiple principalement, et à l'intérieur d'un même symbole) induite (Cf figure 9).

Cependant, si il y a des trajets non résolus (ou encore de codes ayant des auto-corrélations médiocres) le point d'équilibre stable de la boucle peut être influencé par les autres trajets, ce qui entraînerait un biais d'estimation, comme le montre l'expression de la S-courbe:

$$
\begin{aligned}
& S_{P}^{\left(l^{\prime}\right)}\left(\varepsilon_{l^{\prime}}\right)=A^{2} \cdot T_{s} \cdot \rho_{l} \cdot \gamma_{k^{\prime} k^{\prime}}(0) \cdot \sin \left(\varepsilon_{l^{\prime}}\right) \\
& +\underbrace{\operatorname{Im}\left\{A^{2} \cdot T_{s} \cdot \sum_{l \neq l^{\prime}} \rho_{l} \cdot e^{j\left(\theta_{l}-\hat{\theta}_{l^{\prime}}\right)} \gamma_{k^{\prime} k^{\prime}}\left(\tau_{l^{\prime}}-\tau_{l}\right)\right\}}_{I E T}
\end{aligned}
$$

Dans ce cas en effet, $S_{P}^{\left(l^{\prime}\right)}(0) \neq 0$, à cause du terme d'interférence entre les trajets "IET", indiqué en (29). Le point d'équilibre stable de la boucle \# $l^{\prime}$ serait alors donné par: $\hat{\theta}_{e q}=\theta_{l^{\prime}}-\varepsilon_{e q}$, où le biais $\varepsilon_{e q}$ est tel que $S_{P}^{\left(l^{\prime}\right)}\left(\varepsilon_{e q}\right)=0$.

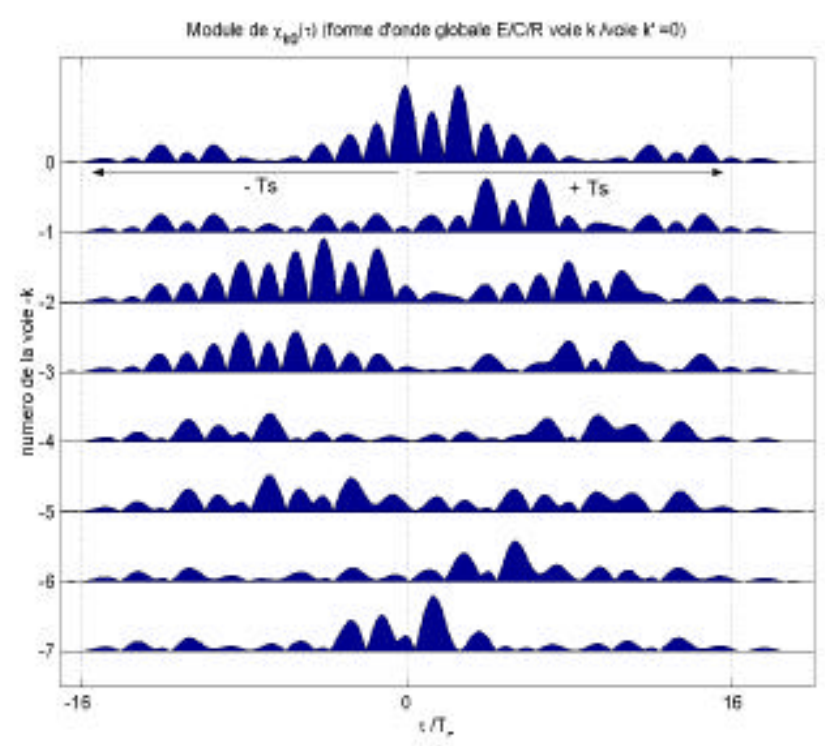

Fig. 9. Exemple de formes d'ondes $E / C / R \chi_{k k^{\prime}}(\tau)$ en CDMA avec 2 trajets

Illustration : la figure 9 présente les modules de 8 ( $k=0$ ...7) des 16 formes d'ondes “émission-canal-réception", $\chi_{k k^{\prime}}(\tau)$, relatives à la voie désirée \# $k^{\prime}=0$, obtenues avec un canal à 2 trajets espacés de $\Delta \tau=2,5 . T_{c}$, de même amplitude, mais déphasés de $\Delta \theta=\pi / 6$. Elles mettent en évidence, en comparaison aux courbes obtenues avec 1 seul trajet (figure 5), l'apparition d'interférence entre voies, surtout au retard nul, et une légère interférence entre symboles $\left(\mathrm{en}+\mathrm{ou}-T_{s}\right)$.

\section{3. en OFDM : une estimation par sous- porteuse?}

En présence de trajets multiples, le récepteur conventionnel OFDM (discuté en section 3.2.3) réalise toujours un échantillonnage synchrone à un seul point par symbole. On ne cherche donc pas ici à recombiner de manière cohérente les trajets, qui de toute manière ne sont pas résolus (vis à vis cette fois de $T_{s}$ ) puisqu'on ne bénéficie pas de l'aspect large-bande. Mais on bénéficie par contre du maintien de l'orthogonalité des formes d'ondes au travers du canal. En effet, les termes d'IES et d'IEV sont d'après (24) des combinaisons linéaires des coefficients:

$$
\chi_{k k^{\prime}}\left(t_{0}+i \cdot T_{s}\right)=\sum_{l=0}^{L-1} \alpha_{l}(t) \cdot \beta_{k k^{\prime}}\left(t_{0}+i \cdot T_{s}-\tau_{l}\right), \quad i \in Z
$$

Pour un étalement du canal $\Delta \tau=\tau_{L-1}-\tau_{0}$ inférieur au temps de garde, $T_{g}$, il est facile de vérifier qu'avec un délai d'échantillonnage $t_{0}$ choisi égal à celui du premier trajet, $t_{0}=\tau_{0}$, ou bien encore choisi dans une plage à gauche, telle que $\left.\left.t_{0}-\tau_{0} \in\right]-T_{g}+\Delta \tau ; 0\right]$, tous les coefficients mis en jeux (hormis l'utile, correspondant à $k=k^{\prime}$ et $i=0$ ) sont 
nuls, et qu'il n'y a donc pas d'interférence. En effet, d'après la discussion initié en 3.2.3 sur le support limité de $\beta_{k k^{\prime}}(\tau)$ et le maintien de l'orthogonalité (20) sur toute une plage grâce au préfixe cyclique, on déduit que le coefficient qui porte le symbole désiré s'évalue à partir de $T_{s} \beta_{k^{\prime} k^{\prime}}\left(t_{0}-\tau_{l}\right)=e^{j 2 \pi f_{k^{\prime}}\left(_{0}-\tau_{l}\right)}$ puisque $\left.\left.\left(t_{0}-\tau_{l}\right) \in\right]-T_{g} ; 0\right], \forall l$. Ainsi:

$$
\chi_{k^{\prime} k^{\prime}}\left(t_{0}\right)=\frac{1}{T_{s}} e^{j 2 \pi f_{k^{\prime}} t_{0}} \cdot \underbrace{\sum_{l=0}^{L-1} \alpha_{l}(t) \cdot e^{-j 2 \pi f_{k^{\prime}} \tau}}_{H_{k^{\prime}}}
$$

Ce coefficient n'est autre que, au déphasage $2 \pi f_{k}, t_{0}$ près, la Transformée de Fourier (TF) de la R.I. du canal analogique de propagation $h(\tau)$, prélevée à la fréquence $f_{k^{\prime}}=k^{\prime} \cdot \frac{W}{N}$, et que l'on notera: $H_{k^{\prime}}$. D'où le simple modèle sans interférence obtenue en sortie de la TFD du récepteur OFDM :

$$
z_{k^{\prime}[m]}=a_{k^{\prime}[m]} \cdot e^{j 2 \pi f_{k^{\prime}} t_{0}} \cdot H_{k^{\prime}}+\eta_{k^{\prime}[m]}
$$

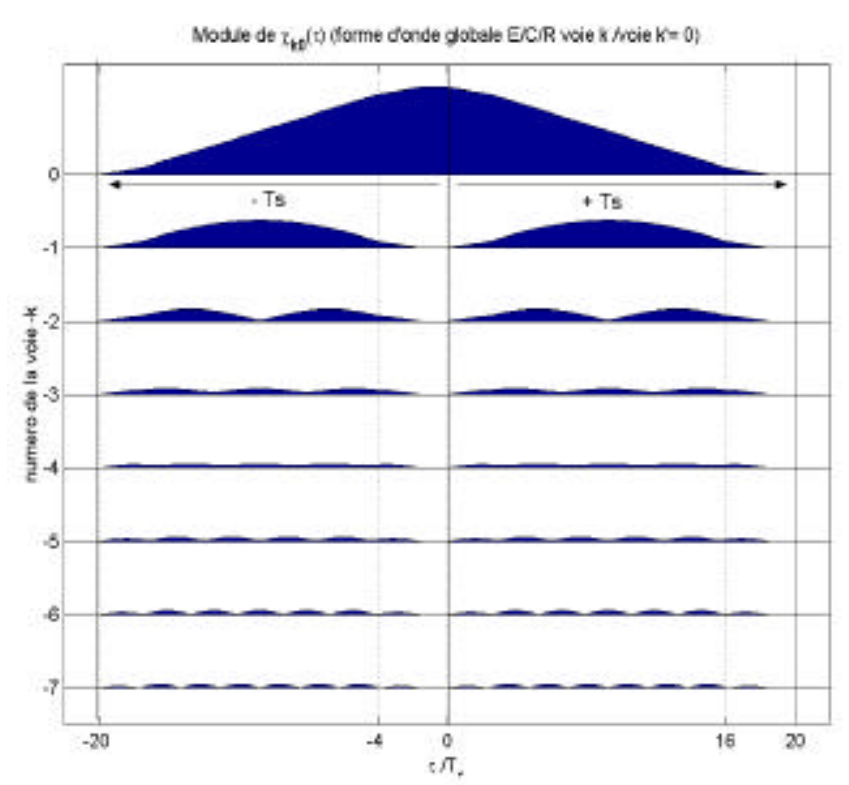

Fig. 10 : Exemple de formes d'ondes E/C/R $\chi_{k k^{\prime}}(\tau)$ en $\operatorname{OFDM}(N=16 v=4)$ avec 2 trajets

Illustration : la figure 10 présente les modules de 8 $(k=0 \ldots 7)$ des 16 formes d'ondes "émission-canalréception', $\chi_{k k^{\prime}}(\tau)$, relatives à la voie désirée $\# k^{\prime}=0$, obtenues avec un canal à 2 trajets espacés de $\Delta \tau=2,5 . T_{c}$, de même amplitude, déphasés de $\Delta \theta=\pi / 6$. Elles mettent en évidence, comparativement aux courbes obtenues en CDMA (figure 9), l'absence d'interférence tant que l'écart de délai entre les trajets est inférieur au temps de garde (avec échantillonnage idéal).

Ainsi la boucle de phase sur la voie $\# k^{\prime}$ va estimer la phase $\theta_{\text {des }}=\operatorname{Arg}\left\{\chi_{k^{\prime} k^{\prime}}\left(t_{0}\right)\right\} \quad$ (correspondant à $\theta_{e q}$ (27)) affectant les symboles de la voie \# $k^{\prime}$, qui s'exprime par:

$$
\theta_{\text {des }}=2 \pi f_{k^{\prime}} t_{0}+\operatorname{Arg}\left\{H_{k^{\prime}}\right\}
$$

Cette phase est égale (au déphasage $2 \pi f_{k}, t_{0}$ ) à la phase de la fonction de transfert analogique prélevée sur la sousporteuse visée. Elle correspond donc à la phase du "macrotrajet', équivalent pour la voie $\# k^{\prime}$, résultant de la superposition des $L$ trajets non-résolus.

L'estimation se fera sans biais, et avec une qualité d'estimation $\varepsilon=\theta_{d e s}-\hat{\theta}$ comparable au cas mono-trajet (absence d'interférence), avec la S-courbe :

$$
S_{P}(\varepsilon)=A^{2} .\left|H_{k^{\prime}}\right| \cdot \sin (\varepsilon)
$$

Finalement en OFDM, la boucle à remodulation donnera de très bonnes performances, comparables à celles obtenues dans le cas mono-trajet mono-voie. Son utilisation pose tout de même le problème du grand nombre $(K)$ de termes de phase à estimer, puisque une boucle ne réalise l'estimation que pour une sous-porteuse $\# k^{\prime}$ donnée. Le problème complet revient à estimer la phase (ou l'amplitude complexe lorsque le module intervient dans la modulations de base, autre que QPSK) de la fonction de transfert du canal pour les différentes fréquences. Néanmoins, on pourra exploiter le fait que les $K$ coefficients complexes, $H_{k^{\prime}}$, ne résultent que de quelques $(2 L)$ paramètres que sont les amplitudes complexes et les retards des trajets, pour faire l'estimation globale du canal à partir de seulement quelques sous-porteuses pilotes bien réparties (du style une par bande de cohérence du canal).

\section{Amélioration de l'algorithme standard en CDMA pour des trajets non-résolus}

\subsection{Discussion}

On se focalise dorénavant sur le cas CDMA, dans une situation où l'on doit suivre de rapides variations de phases du canal, avec des trajets qui ne sont pas tous résolus. Nous avons vu que le détecteur d'erreur de phase (DEP) standard avait un fonctionnement dégradé avec des trajets nonrésolus, car alors la sortie du DEP relative à 1 trajet pouvait dépendre fortement des autres trajets (avec en plus un bruit de boucle augmenté à cause de l'IEV induite). Il serait donc intéressant de pouvoir réduire l'effet des autres trajets, qui se traduit par un terme d'IET dans la S-courbe donnée en équation (29).

Une manière simple d'y parvenir pourrait consister à reconstruire le terme d'IET et à le soustraire à la sortie du DEP de la boucle de phase. Cependant, une telle technique n'est pas forcément adéquate pour un contexte de rapides variations de canal, car la mise à jour du terme de correction nécessite un parfait suivi des phases mais aussi des amplitudes des trajets. Nous allons décrire ici une stratégie différente [6]: elle exploite le fait que les trajets sont invariants sur la durée du "slot", afin de rendre une "correction" insérée dans la boucle de phase, Indépendante des variations des Phases ou des Amplitudes des trajets. Cette "correction", basée sur le concept de préfiltrage ne nécessite aucune adaptation, et permet de maintenir à zéro le terme d'IET lorsque les délais sont fixes. 


\subsection{Introduction d'un préfiltre dans la boucle}

On s'intéresse par défaut à la boucle associée au trajet $\# l^{\prime}$. Conceptuellement, l'introduction d'un préfiltre de R.I. $p(\tau)$ dans la boucle de phase $\# l^{\prime}$ revient à séparer le chemin de traitement de la synchronisation de phase, de celui de la détection. On obtient alors un schéma (figure 11) modifié par rapport au schéma standard (figure 3).

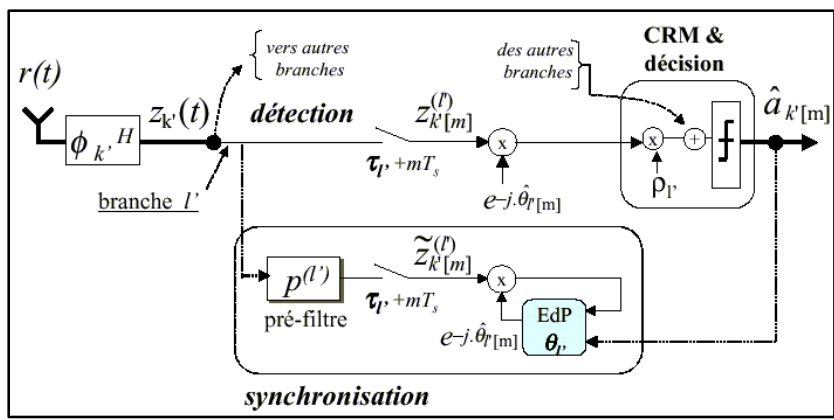

Fig. 11: structure (formelle) du récepteur utilisant un préfiltre dans la boucle de synchronisation ( $l^{\text {eme }}$ branche)

Le signal d'erreur est toujours construit selon (10), mais en remplaçant l'échantillon $z_{k^{\prime}}$ (.) par l'échantillon préfiltré $\tilde{z}_{k^{\prime}}\left(m T_{s}+\tau_{l^{\prime}}\right)$. Ainsi, formellement, c'est toujours (au travers du canal) la forme d'onde $E / R \quad \gamma_{k^{\prime} k^{\prime}}(\tau)$ qui conditionne la détection, mais c'est une forme d'onde $\mathrm{E} / \mathrm{R} / \mathrm{P} \tilde{\gamma}_{k^{\prime} k^{\prime}}(\tau)$ corrigée par le préfiltre qui conditionne la synchronisation, avec $\tilde{\gamma}_{k^{\prime} k^{\prime}}(\tau)=\left(\gamma_{k^{\prime} k^{\prime}} * p\right)(\tau)$. Cela donne des degrés de liberté dans l'optimisation des 2 tâches.

Plusieurs critères sont possibles pour calculer les coefficients du préfiltre. Dans tous les cas, pour annuler le biais, il faut contraindre la nouvelle S-courbe, donnée en (29) en remplaçant $\gamma_{k k^{\prime}}$ par $\tilde{\gamma}_{k k^{\prime}}$, à zéro: $\tilde{S}_{P}^{\left(l^{\prime}\right)}(0)=0$ (Cf figure 13.b). Cette condition nécessite, pour un coefficient $\tilde{\gamma}_{k k^{\prime}}(0)$ réel, de forcer à zéro le terme d'IET. Si on veut de plus que cette annulation soit indépendante des variations de phases ou d'amplitudes, il faut contraindre la forme d'onde corrigée $(E / R / P)$ de telle sorte qu'elle présente une amplitude nulle aux positions de chaque trajet adjacent, soit : $\quad \tilde{\gamma}_{k^{\prime} k^{\prime}}\left(\tau_{l^{\prime}}-\tau_{l}\right)=0, \forall l^{\prime} \neq l$ (Cf figure 12.b). Le calcul des coefficients du préfiltre est détaillé dans [6], et la solution rappelée dans [8]. Dans les illustrations ci-dessous, le préfiltre utilisé a une durée d'environ $T_{s} / 3$.

L'insertion d'un préfiltre exploitant la non-variation des délais des trajets adjacents (en mode burst) peut ainsi apporter une solution à la poursuite de variations rapides de phase, qui ne souffre plus de problème de biais d'estimation ( $\mathrm{Cf}$ figure 15). Une telle stratégie peut-être utilisée tant que le nombre de trajets reste faible. La poursuite améliorée des phases pourrait être couplée à la poursuite des variations des amplitudes. Elle peut aussi servir d'autres détecteurs plus sophistiqués que le Rake, du moment que ceux-ci ont une utilisation explicite des phases (et amplitudes) des trajets.

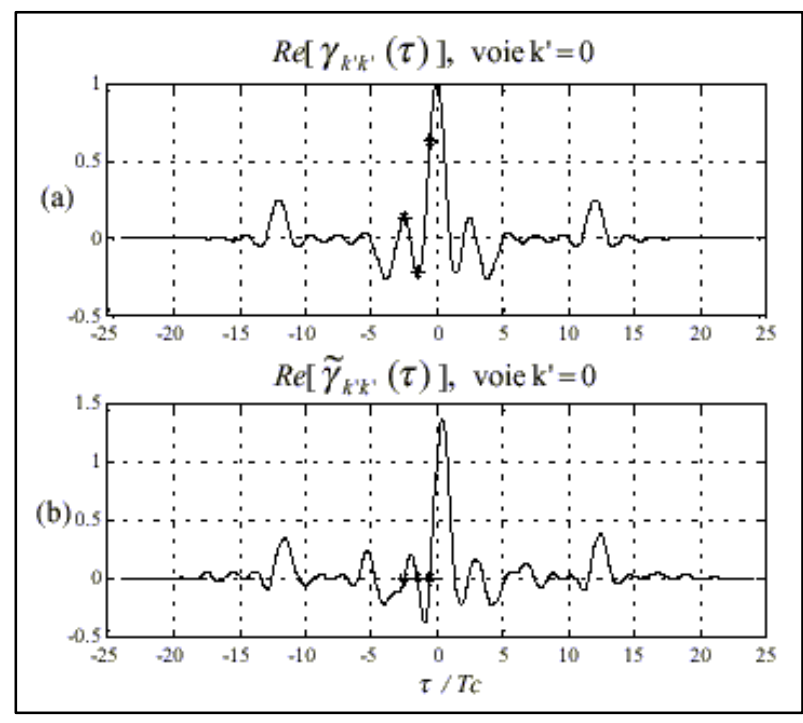

Fig 12. (a) forme d'onde E/R désirée $\gamma_{k^{\prime} k^{\prime}}(\tau)$ et $(b)$ version préfiltrée $E / R / P \tilde{\gamma}_{k^{\prime} k^{\prime}}(\tau)$

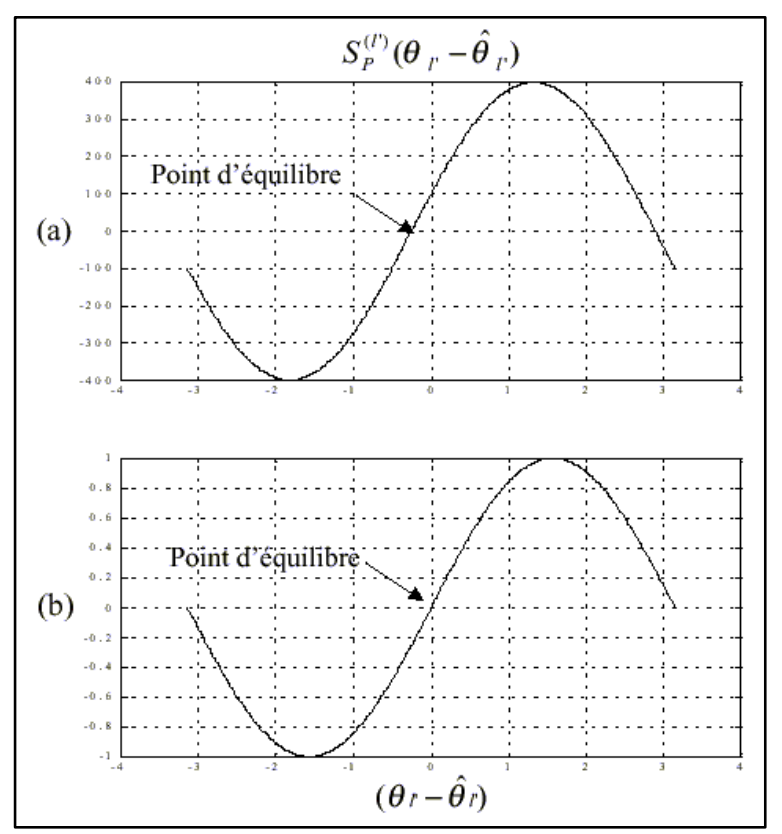

Fig. 13 : S-courbe obtenue: (a) sans préfiltre et (b) avec préfiltre

Illustration de l'annulation de biais: La figure 12 illustre l'effet du préfiltre sur la partie réelle de la fonction d'équipement $E / R$ désirée initiale, $\gamma_{k^{\prime} k^{\prime}}$, pour un canal en lien descendant composé de 4 trajets avec les délais relatifs de $0,0.5 T_{c}, 1.5 T_{c}$, et $2.5 T_{c}$, et des amplitudes de 0 $d B,-0.9 d B,-4.9 d B$ et $-8 d B$. Sans le préfiltre, la partie (a) de la figure 12 montre que la fonction E/R initiale, construite à partir d'une séquence de Walsh-Hadamard (embrouillée), n'est pas très performante du point de vue de la synchronisation : les valeurs d'auto-corrélations nonnégligeables aux retards chips non nuls, induiront clairement de l'interférence entre trajets importante avec de tels trajets. Pour le trajet désiré $l^{\prime}=0$, la partie (b) de la figure 12 montre l'effet du préfiltre, qui force à zero la forme d'onde préfiltrée $(E / R / P)$ aux points $\tau_{0}-\tau_{1}, \tau_{0}-\tau_{2}$ et $\tau_{0}-\tau_{3}$ (indiqués par une '*' sur la figure). La figure 13 montre la S-courbe du DEP optimisé (partie (b)), en comparaison avec la S-courbe du DEP standard (a). Pour le DEP standard, on note que le passage à zéro de la $S$ - 
courbe ne se fait pas à l'origine, mais à $\left(\theta_{0}-\theta_{0_{e q}}\right) \approx-025 \mathrm{rad}$. Il y aura en conséquence un biais statique sur l'estimation de phase (pour un scénario statique du canal). Pour la S-courbe du DEP optimisé grâce au préfiltre, le passage à zéro est ramené à l'origine, permettant une estimation sans biais.

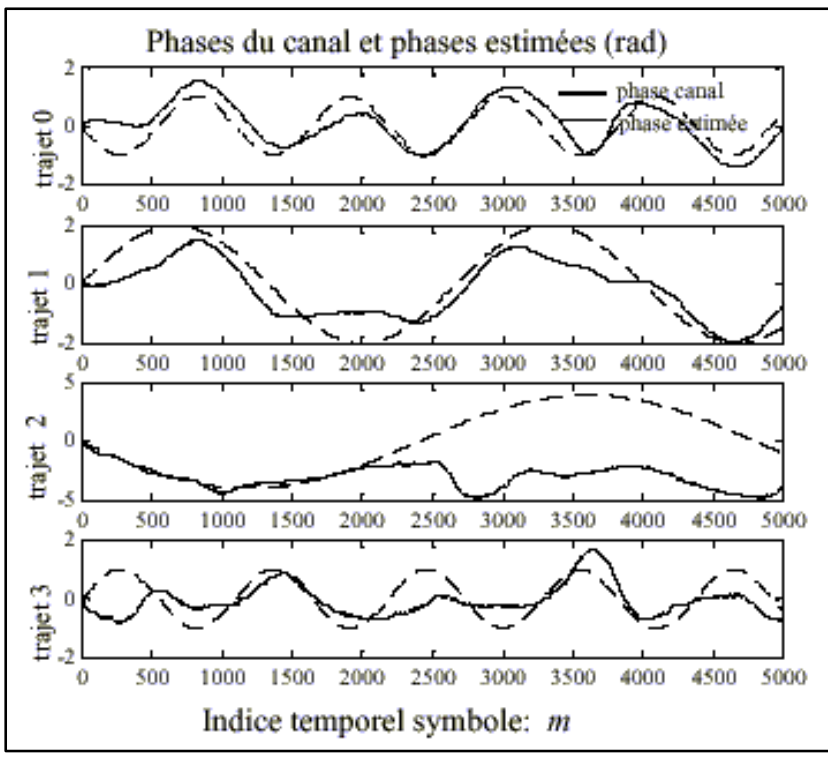

Fig 14 : Poursuite des phases avec la boucle du $2^{\circ}$ ordre standard (sans préfiltre), $E_{b} / N_{0}=20 \mathrm{~dB}$

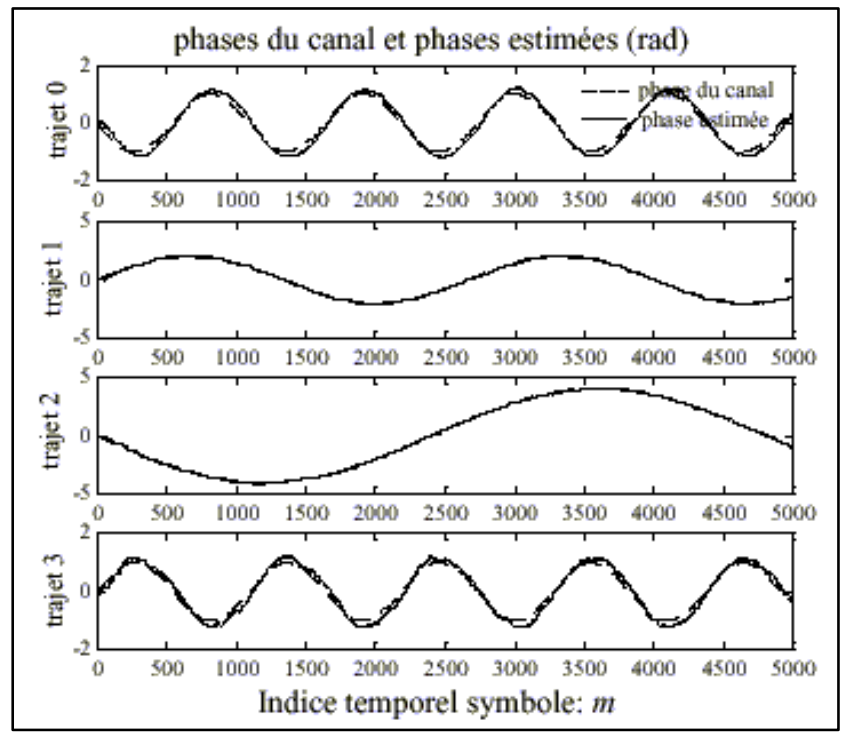

Fig. 15 : Poursuite des phases avec la boucle du $2^{\circ}$ ordre améliorée (avec préfiltre), $E_{b} / N_{0}=20 \mathrm{~dB}$

Illustration en dynamique: Les figures 14 et 15 illustrent respectivement les performances en poursuite des boucles standard et améliorée, supposées bien initialisées, pour un scénario avec $K=5$ utilisateurs, le canal de 4 coefficients mentionné, un rapport $E_{b} / N_{0}$ de $20 \mathrm{~dB}$. Nous montrons les phases poursuivies et les vraies phases du canal de chaque trajet. La simulation est réalisée avec des modèles de phases sinusoïdaux $\theta_{l}(t)=\Delta \theta_{l} \sin \left(2 \pi f_{g}^{(l)} t\right)$, de fréquence de gigue $f_{g}$ égales à $-220 \mathrm{~Hz}, 90 \mathrm{~Hz},-50 \mathrm{~Hz}, 220 \mathrm{~Hz}$ pour les trajets 1 à 4, ce qui permet de "balayer" différents scénarios de Doppler pouvant se produire en mode TDD de l'UMTS, pour une vitesse du mobile de $v_{m}=120 \mathrm{Km} / \mathrm{h}$.
Les coefficients des filtres de boucle $f_{B}^{(l)}$ sont choisis (Cf annexe A.1) pour obtenir des boucles du second ordre, de facteur d'amortissement $\zeta=0.7$ et de fréquence propre $f_{n}=500 \mathrm{~Hz}$ pour chaque boucle, ce qui permettrait en pratique de suivre de rapides variations aléatoires de phase. On voit qu'avec un tel scénario de trajets nonrésolus, la boucle standard est en échec (sauf peut-être pour le trajet \# 0 avec un biais évolutif, ou dans la portion initiale du trajet \# 2 ), alors que la boucle améliorée grâce au préfiltre réalise une poursuite tout à fait correcte.

\section{Conclusion générale}

Nous sommes partis d'un algorithme standard (en contexte mono-trajet et mono-utilisateur) de poursuite de phase à l'aide des décisions et avons discuté de son adéquation en contexte à $L$ trajets et $K$ voies. Malgré un formalisme de modulation identique, l'utilisation possible de l'algorithme (pour un canal multi-trajet identique décrit dans une bande $W$ ) prend un aspect très différent selon les caractéristiques des formes d'ondes.

En CDMA, on profite au maximum de la résolution temporelle que permet l'importante largeur de bande $W$, en vue de distinguer les trajets et de faciliter une recombinaison cohérente. L'algorithme standard peut être utilisé pour poursuivre directement (1 boucle par trajet) les phases des trajets de propagation, mais avec une variance d'estimation qui se dégrade avec le nombre d'utilisateurs en raison d'une orthogonalité brisée par les trajets multiples, et un problème de biais d'estimation qui peut apparaître si les trajets ne sont pas bien résolus. Nous avons présenté une possibilité de résoudre ce dernier problème dans le cas où les délais ne varient pas, grâce à l'insertion d'un préfiltre dans la boucle de phase. Cela permet de donner des degrés de liberté à la tâche de synchronisation, relativement à celle de détection, afin d'obtenir une indépendance vis à vis des trajets adjacents, et de tolérer l'utilisation de codes peu performants du point de vue de la synchronisation mais intéressants pour la détection (comme les codes à base des séquences de Walsh-Hadamard, très souvent utilisés).

En OFDM, on a un comportement complètement différent puisque les formes d'ondes sont bande-étroite (de largeur $W /(N+v)$ par voie), ce qui ne permet pas de distinguer les trajets. Le récepteur conventionnel s'affranchit par contre complètement du problème d'interférence par la suppression du préfixe cyclique introduit à l'émission : tout se passe comme si chaque voie de symboles était affectée par un canal mono-trajet d'amplitude complexe différente d'une voie (sous-porteuse) à l'autre. L'algorithme standard est alors capable de bien estimer la phase "résultante" et pourra être associé à une estimation d'ensemble des paramètres, afin de ne pas être répété pour chacune des $K$ sous-porteuses. En contre-partie, rappelons qu'une telle stratégie bande-étroite avec insertion / suppression d'un préfixe cyclique (sans filtre adapté au canal en réception) rend extrêmement sensible aux évanouissements (Cf [5], [2]), qui se produisent lorsque le macro-trajet d'une sousporteuse \# $k$ résulte d'une combinaison destructive des $L$ trajets $\left(\left|H_{k}\right|=0\right)$ à la fréquence de cette sous-porteuse. 


\section{A. ANNEXES}

\section{A.1. Annexe: performance d'une boucle de phase}

De manière générale, d'un point de vue statique (pour $\theta$ constant), le signal d'erreur d'une boucle de phase peut se décomposer en un terme certain $\left(S_{P}\right)$, fonction de l'erreur de phase $\varepsilon_{[m]}=\theta_{[m]}-\hat{\boldsymbol{\theta}}_{[m]}$, appelé S-courbe, et d'un terme aléatoire centré $\left(N_{P}\right)$, qui représente le bruit de boucle:

Par définition:

$$
v_{\varepsilon[m]}=S_{P}(\varepsilon)+N_{P[m]}
$$

- la S-Courbe est obtenue en calculant l'espérance du signal d'erreur $v_{\varepsilon}$ conditionnellement à des valeurs fixes de l'erreur de phase $\left(\varepsilon_{[m]}=\varepsilon\right)$ :

$$
S_{P}(\theta-\hat{\theta}) \stackrel{\Delta}{=} E\left\{v_{\varepsilon[m]} \mid \theta-\hat{\theta}\right\}
$$

Expérimentalement, $S_{P}(\varepsilon)$ est obtenu en boucle ouverte en mesurant la moyenne temporelle du signal d'erreur, pour une valeur connue arbitraire fixe de $\theta$ à l'entrée, et une valeur fixe de $\hat{\theta}$ réglée à $\hat{\theta}=\theta-\varepsilon$.

- $\quad$ le bruit de boucle est donc : $N_{P[m]}=v_{\varepsilon[m]}-S_{P}(\varepsilon)$, il est dans les cas idéaux indépendant de $\varepsilon$.

Dans un contexte de faibles variations d'erreur de phase (régime de poursuite), les performances d'une boucle de phase se déduisent des caractéristiques du détecteur d'erreur de phase. On peut en effet [1] linéariser la $S$ courbe autour de son point d'équilibre stable, $\varepsilon_{e q}$, obtenu (condition nécessaire) pour $S\left(\varepsilon_{e q}\right)=0$. On a alors :

$$
S(\varepsilon)=D \cdot\left(\varepsilon-\varepsilon_{e q}\right)
$$

où $D$ est la pente (positive) autour du point d'équilibre stable. Il est souhaitable que $\varepsilon=0$ soit un point d'équilibre stable, c'est à dire que $S(0) \stackrel{\Delta}{=} E\left\{v_{\varepsilon[m]} \mid \varepsilon=0\right\}=0$. Notons que dans le cas contraire, $\varepsilon_{e q}$ est différent de 0 et il correspond à un biais d'estimation, amenant en moyenne $(E\{\hat{\theta}\})$ à une estimation $\hat{\theta}_{e q}=\theta-\varepsilon_{e q}$ au lieu de $\hat{\theta}_{e q}=\theta$.

Avec le modèle linéaire (et avec l'approximation d'un bruit de boucle non corrélé), la variance de l'erreur d'estimation, pour une phase $\theta$ statique, est donnée par:

$$
\sigma_{\varepsilon_{N p}}^{2}=E\left\{\left(\varepsilon-\varepsilon_{e q}\right)^{2}\right\}=2 \cdot B_{p} \cdot T_{s} \cdot \frac{\sigma_{N_{P}}^{2}}{D^{2}}
$$

où $\sigma_{N_{P}}^{2}$ est la variance du bruit de boucle, et $B_{p}, T_{s}$ est la bande de boucle, qui se calcule à partir des éléments du filtre de boucle. Pour obtenir une boucle de fonction de transfert $H_{B}(z)=\frac{\hat{\Theta}(z)}{\Theta(z)}$ du second ordre, on peut prendre un filtre de boucle de R.I. $f_{B}$ donné par:

$$
f_{B}(z)=K_{1}+\frac{K_{2}}{1-z^{-1}}
$$

où $K_{1}$ et $K_{2}$ sont les coefficients du filtre de boucle, qui permettent de régler le facteur d'amortissement, $\zeta$, et la fréquence propre, $f_{n}\left(<<1 / T_{s}\right)$, de la boucle, selon :

$$
\begin{gathered}
f_{n} \cdot T_{s}=\frac{\sqrt{D K_{2}}}{2 \pi} \\
\zeta=\frac{\left(K_{1}+K_{2}\right) \sqrt{D}}{2 \sqrt{K_{2}}}
\end{gathered}
$$

La bande de boucle (du $2^{\text {nd }}$ ordre) est donnée [1] par :

$$
B_{p} \cdot T_{s}=\frac{2 K_{2}+K_{1} D \cdot\left(2 K_{1}+K_{2}\right)}{2\left[4 K_{1}-K_{1} D \cdot\left(2 K_{1}+K_{2}\right)\right]} \approx 2 \pi f_{n} T_{s}\left(\zeta+\frac{1}{4 \zeta}\right)
$$

D'un point de vue dynamique (pour $\theta$ variable), l'erreur d'estimation est aussi dûe au mauvais suivi des variations de $\theta$, lorsque la fréquence propre $f_{n}$ est insuffisante. La variance d'erreur supplémentaire dans l'approximation linéaire est donnée par:

$$
\sigma_{\varepsilon_{\theta}}^{2}=\int_{-1 / 2 T_{s}}^{+1 / 2 T_{s}} \Gamma_{\theta}(f) \cdot\left|1-H_{B}\left(e^{j 2 \pi f T_{s}}\right)\right|^{2} d f
$$

où $\Gamma_{\theta}(f)$ est la densité spectrale de la phase variable $\theta$.

Ainsi, lorsque $\theta$ varie et en présence de bruit additif, un compromis doit être fait dans le choix de $f_{n}$, selon (37) d'une part et (33) d'autre part.

\section{References}

[1] U.Mengali, A.N. D'Andrea, "Synchronization techniques for digital receivers", Plenum press, 1997.

[2] Z. Wang, G.B. Giannakis, "Wireless multicarrier communications: where Fourier meets Shannon", IEEE Signal Processing Magazine, vol 47, n³, pp 29-48, May 2000.

[3] T. Keller, L. Piazzo, P. Mandarini, L. Hanzo, "Orthogonal Frequency Division Multiplex Synchronization Techniques for Frequency-Selective Fading Channels', IEEE Journal on Selected areas in comm., vol 19, n6, pp. 999-1008, june 2001.

[4] J.J. van de Beek, M. Sandell, P.O. Börjesson, "ML estimation of timing and frequency offset in OFDM systems", IEEE Trans on Signal Processing, vol 45, n7, pp 1800-1805, July 1997.

[5] B. Muquet, Z. Wang, G.B. Giannakis, M. de Courville, P. Duhamel, "Cyclic-prefixing or Zero-Padding for wireless multicarrier transmissions? ', IEEE Trans. Communications, vol 50, $\mathrm{n}^{\circ} 12$, pp 2136-2148, December 2002.

[6] E. Simon, K. Raoof, L. Ros, "Synchronization over rapidly timevarying multipath channels for CDMA downlink receiver in time-division mode", EUSIPCO, sept. 2004, Vienna, Austria.

[7] L. Ros, G. Jourdain, M. Arndt, "Interpretations and Performances of linear receiving in downlink TD-CDMA and Multi-Sensor extension", Annals of telecommunications, vol 56, $\mathrm{n}^{\circ} 5-6$, pp 275-290, May-June 2001.

[8] L. Ros, E. Simon, Y. Nasser, "Synchronisation de phase relative aux variations d'un canal à trajets multiples pour un récepteur radio-mobile à modulation linéaire opérant en mode Burst”, recueil des journées d'études SEE, Paris, Juin 2004. 